\title{
A DFT Investigation of the Addition Reaction of Monomeric Lithium Enolate Derived from Propiophenone to Propene Oxide: Examination of the Possible Transition Structures
}

\author{
Christian Silvio Pomelli, \\ Dipartimento di Chimica e Chimica Industriale, Università di Pisa, Via Risorgimento 35, 56126 Pisa \\ Anna Maria Bianucci \\ Dipartimento di Scienze Farmaceutiche, Università di Pisa, Via Bonanno 6, 56126 Pisa \\ Paolo Crotti*, Lucilla Favero* \\ Dipartimento di Chimica Bioorganica e Biofarmacia, Università di Pisa, Via Bonanno 33, 56126 Pisa, \\ Italy \\ lucillaw@farm.unipi.it
}

\section{Supporting Information}

\section{List of contents}

-Table 2 (extended)

-Table 3

-Table 4

-Table 5

-Experimental details of the reaction of enolate 1 with epoxide 2.

-Computational Data, that include Z-matrices, absolute energies and other thermochemical data of the all optimized structures: TS3Gc, -Ac, -Go, -Ao, TS4Gc, -Ac, -Go, -Ao, complexes 7a and 7b, enolate 1, epoxide 2, TS', TS' ' and corresponding intermediate I, and reaction products 3a-d and 4ac. 
TABLE 2 (Extended). $\Delta E e^{\#}, \Delta E e, \Delta G^{\#}, \Delta G\left(\mathrm{kcal} \mathrm{mol}^{-1}\right)$, for Each Reaction Pathway. Vacuum MP2/631+G(d), Vacuum and IEF-PCM Solvent Model B3L YP/6-31+G(d) Results.

\begin{tabular}{|c|c|c|c|c|c|c|c|c|c|c|c|c|}
\hline Entry & Reaction pathway & $\Delta E e^{\# a}$ & $\Delta E e^{\# b}$ & $\Delta E e^{\# c}$ & $\Delta E e_{r e l}^{\#}{ }_{r, d}^{b}$ & $\Delta E e^{\#}{ }_{r e l}^{c, d}$ & $\Delta E e^{a}$ & $\Delta E e^{b}$ & $\Delta E e^{c}$ & $\Delta G^{\# b, e}$ & $\Delta G^{b, e}$ & $\Delta G_{r e l}^{\#} b, e, f$ \\
\hline 1 & 7a-->TS3Gc-->3a & +38.18 & +35.60 & +36.42 & 0.00 & 0.00 & -31.34 & -26.97 & -26.73 & +39.19 & -19.81 & 0.00 \\
\hline 2 & 7a-->TS3Ac-->3b & +39.29 & +40.22 & +40.39 & +4.62 & +3.97 & -15.00 & -9.14 & -14.46 & +44.98 & -4.10 & +5.79 \\
\hline 3 & 7b-->TS3Go-->3d & +26.86 & +25.81 & +23.84 & +6.65 & +6.17 & +5.00 & +5.28 & -2.67 & +27.79 & +8.78 & +5.87 \\
\hline 4 & 7a-->TS3Ao-->3c & +47.54 & +46.83 & +38.45 & +11.23 & +2.03 & -29.43 & -26.10 & -26.05 & +49.70 & -19.46 & +10.51 \\
\hline 5 & 7a-->TS4Gc-->4a & +47.48 & +42.74 & +44.08 & +7.14 & +7.66 & -27.55 & -22.92 & -23.24 & +46.47 & -16.45 & +7.28 \\
\hline 6 & $7 a-->T S 4 A c-->4 b$ & +39.43 & +40.30 & +40.48 & +4.70 & +4.05 & -18.11 & -11.94 & -17.00 & +45.26 & -7.40 & +6.07 \\
\hline 7 & 7b-->TS4Go-->4c & +26.79 & +25.84 & +23.76 & +6.68 & +6.10 & -1.36 & +0.59 & -5.14 & +27.66 & +5.03 & +5.74 \\
\hline 8 & 7a-->TS4Ao-->4b & +49.42 & +48.08 & +39.54 & +12.47 & +3.12 & -18.11 & -11.94 & -17.00 & +51.17 & -7.40 & +11.98 \\
\hline 9 & 7a--> TS' $-->\mathbf{I}^{g}$ & +23.76 & +20.96 & +18.91 & & & +17.73 & +16.53 & +18.41 & +18.38 & +15.09 & \\
\hline 10 & I-->TS"-->7b & +0.25 & +1.51 & +1.11 & & & -1.43 & -0.09 & +0.35 & +3.75 & +2.18 & \\
\hline 11 & isolated reag.-->7a & ------ & ------ & ------ & ------ & & -25.18 & -21.85 & -9.71 & ------ & -26.93 & \\
\hline 12 & isolated reag.-->7b & ------ & ------ & ------ & ------ & & -9.12 & -5.41 & +9.04 & ------ & -9.67 & \\
\hline
\end{tabular}

${ }^{\bar{a}} \mathrm{MP} 2 / 6-31+\mathrm{G}(\mathrm{d})$ vacuum results. ${ }^{b}$ B3LYP/6-31+G(d) vacuum results. ${ }^{c}$ B3LYP/6-31+G(d) IEF-PCM results. ${ }^{d}$ The difference of the electron energy $E e^{*}$ value of the TS of entry 1 (TS3GC) and the electron energy $E e^{*}$ value of the TS under examination. ${ }^{e}$ The calculated free energy values in vacuum for all structures (reactants, products and TSs), were corrected by adding the ZPE and taking away E-TS rotational and translational terms. ${ }^{f}$ The difference of the corrected free energy $G^{\#}$ value of the TS of entry 1 (TS3GC) and the corrected free energy $G^{\#}$ value of the TS under examination. ${ }^{8} \mathbf{I}=$ Intermediate, see Schemes 3 and 4.

TABLE 3. Second Order Perturbation Analysis of the Fock Matrix in NBO Basis of Open TSs (TS4Go, TS4Ao, TS3Go and TS3Ao) and Complex 7a and 7b. (Threshold for $E 2=$ $2.00 \mathrm{kcal} \mathrm{mol}^{-1}$ ).

\begin{tabular}{ccclc}
\hline entry & Structure & Donor NBO & Acceptor NBO $\mathrm{kcal} \mathrm{mol}^{-1}$ \\
\hline & & & & \\
1 & TS4Go & LP(1)O8 & LP*(1)Li11 & 11.26 \\
2 & & BD(1)C7-O8 & LP*(1)Li11 & 4.29 \\
3 & & BD(1)C7-C9 & LP*(1)Li11 & 3.37 \\
4 & & LP(2)O8 & LP*(1)Li11 & 4.86 \\
5 & & CR(1)O8 & LP*(1)Li11 & 2.00 \\
6 & & BD(1)C10-H19 & LP*(1)Li11 & 2.43 \\
7 & & BD(1)C12-C14 & LP*(1)Li11 & 2.20 \\
8 & & BD(1)C12-H27 & LP*(1)Li11 & 5.38 \\
9 & & BD(1)C15-H30 & LP*(1)Li11 & 2.72 \\
& & & & \\
10 & TS4Ao & LP(1)O8 & LP*(1)Li11 & 6.79 \\
11 & & LP(2)O8 & LP*(1)Li11 & 2.75 \\
12 & & LP(2)O8 & RY*(2)Li11 & 2.06
\end{tabular}




\begin{tabular}{|c|c|c|c|c|}
\hline entry & Structure & Donor NBO & Acceptor NBO & $\begin{array}{c}E 2^{a} \\
\mathrm{kcal} \mathrm{mol}^{-1}\end{array}$ \\
\hline 13 & TS3Go & $\mathrm{BD}(1) \mathrm{C} 7-\mathrm{C} 9$ & LP*(1)Li11 & 2.67 \\
\hline 14 & & $\mathrm{BD}(1) \mathrm{C} 7-\mathrm{O} 8$ & LP*(1)Li11 & 3.69 \\
\hline 15 & & BD(1)C10-H19 & LP*(1)Li11 & 2.33 \\
\hline 16 & & LP(1)O8 & LP*(1)Li11 & 13.66 \\
\hline 17 & & $\mathrm{LP}(2) \mathrm{O} 8$ & LP*(1)Li11 & 11.66 \\
\hline 18 & & $\mathrm{LP}(3) \mathrm{O} 8$ & $\mathrm{LP}^{*}(1) \mathrm{Li1} 1$ & 3.56 \\
\hline 19 & & $\mathrm{BD}(1) \mathrm{C} 12-\mathrm{C} 14$ & LP*(1)Li11 & 2.43 \\
\hline 20 & & $\mathrm{BD}(1) \mathrm{C} 12-\mathrm{H} 27$ & LP*(1)Li11 & 4.18 \\
\hline 21 & & $\mathrm{BD}(1) \mathrm{C} 15-\mathrm{H} 30$ & LP*(1)Li11 & 3.79 \\
\hline 22 & TS3Ao & $\mathrm{LP}(1) \mathrm{O} 8$ & LP*(1)Li11 & 6.79 \\
\hline 23 & & $\mathrm{LP}(2) \mathrm{O} 8$ & $\mathrm{LP}^{*}(1) \mathrm{Li} 11$ & 2.75 \\
\hline 24 & & $\mathrm{LP}(2) \mathrm{O} 8$ & $\mathrm{RY}^{*}(2) \mathrm{Li} 11$ & 2.06 \\
\hline 25 & $7 b$ & $\mathrm{BD}(1) \mathrm{C} 7-\mathrm{O} 8$ & LP*(1)Li11 & 3.73 \\
\hline 26 & & $\mathrm{BD}(1) \mathrm{C} 7-\mathrm{C} 9$ & LP*(1)Li11 & 3.99 \\
\hline 27 & & $\mathrm{BD}(2) \mathrm{C} 7-\mathrm{C} 9$ & LP*(1)Li11 & 2.65 \\
\hline 28 & & $\mathrm{LP}(1) \mathrm{O} 8$ & LP*(1)Li11 & 10.10 \\
\hline 29 & & $\mathrm{BD}(1) \mathrm{C} 12-\mathrm{C} 14$ & LP*(1)Li11 & 3.24 \\
\hline 30 & $7 \mathbf{a}$ & $\mathrm{BD}(1) \mathrm{C} 7-\mathrm{O} 8$ & LP*(1)Li11 & 2.76 \\
\hline 31 & & $\mathrm{CR}(1) \mathrm{O} 8$ & LP*(1)Li11 & 2.22 \\
\hline 32 & & LP(1)O8 & LP*(1)Li11 & 12.29 \\
\hline 33 & & $\mathrm{LP}(1) \mathrm{O} 8$ & RY*(3)Li11 & 2.04 \\
\hline 34 & & CR(1)Li11 & $\mathrm{BD}^{*}(1) \mathrm{C} 7-\mathrm{O} 8$ & 3.21 \\
\hline 35 & & $\mathrm{LP}(1) \mathrm{O} 13$ & LP*(1)Li11 & 5.90 \\
\hline 36 & & $\mathrm{LP}(2) \mathrm{O} 13$ & $\mathrm{LP}^{*}(1) \mathrm{Li} 11$ & 6.03 \\
\hline
\end{tabular}

LP: Lone Pair orbital; BD: Bond orbital; CR: Core orbital; RY: Rydberg orbital; *: unoccupied orbital.

${ }^{a} \quad E 2=$ delocalization energy. See NBO 4.M Program Manual (Gaussian Inc.).

TABLE 4. Geometrical Parameters, W Coefficient and $\Delta E e^{\#}\left(\right.$ Solvent), $\Delta G^{\#}\left(V_{\text {acuum}}\left(\mathrm{kcal}^{\mathrm{mol}}{ }^{-1}\right)\right.$, for Transition States TS3Gc, TS3Ac, TS3Go, TS3Ao, TS4Gc, TS4Ac, TS4Go and TS4Ao.

\begin{tabular}{cccccccccc}
\hline Entry & Structure & Angle $\alpha$ & Angle $\beta$ & dih1 & dih2 & $\mathrm{W}$ & $\mathbf{d}(\AA)$ & $\Delta G \#^{a, b}$ & $\left.\Delta^{a}\right) \#^{c}$ \\
\hline 1 & TS3Gc & 99.145 & 80.104 & 71.029 & 132.160 & 0.862 & 3.94 & +39.19 & +36.42 \\
2 & TS3Ac & 152.731 & 82.555 & 61.380 & 152.364 & 1.294 & 5.20 & +44.98 & +40.39 \\
3 & TS4Gc & 115.206 & 79.995 & 70.221 & 120.239 & 1.164 & 3.28 & +46.47 & +44.08 \\
4 & TS4Ac & 152.507 & 83.005 & 59.488 & 151.469 & 1.317 & 4.17 & +45.26 & +40.48 \\
5 & TS3Go & 124.459 & 102.665 & 82.923 & -176.062 & 0.536 & 3.70 & +27.79 & +23.84 \\
6 & TS3Ao & 113.092 & 100.328 & 91.185 & 175.112 & 0.327 & 3.99 & $+22.77(+49.70)^{d}$ & $+28.74(+38.45)^{d}$ \\
7 & TS4Go & 125.043 & 104.834 & -82.965 & 170.425 & 0.627 & 4.81 & +27.66 & +23.76 \\
8 & TS4Ao & 115.371 & 101.195 & -94.970 & -176.389 & 0.390 & 3.44 & $+24.23(+51.17)^{d}$ & $+29.83(+39.48)^{d}$ \\
\hline
\end{tabular}


B3LYP/6-31+G(d) vacuum results. ${ }^{b}$ The calculated free energy values in vacuum for all structures (reactants, products and TSs), were corrected by adding the ZPE and taking away E-TS rotational and translational terms. ${ }^{c}$ B3LYP/6-31+G(d) IEF_PCM results. ${ }^{d}$ See ref.17.

TABLE 5. Absolute Electronic Energies. B3LYP/6-31+G(d) Vacumm Results $\left(E_{e_{v a c}}\right)$, IEF-PCM B3LYP/6-31+G(d) Results $\left(E e_{\text {solv }}\right)$, and Relative Differences $\left(E e_{\text {solv }}-E e_{\text {vac }}\right)$.

\begin{tabular}{|c|c|c|c|c|}
\hline entry & Struct. & $\begin{array}{c}E e_{v a c} . \\
\text { a.u. }\end{array}$ & $\begin{array}{l}E e_{\text {solv. }} \\
\text { a.u. }\end{array}$ & $\begin{array}{l}E e_{\text {solv }}-E e_{v q c} \\
\left(\mathrm{kcal} \mathrm{mol}^{-1}\right)\end{array}$ \\
\hline 1 & TS3Gc & -624.269160 & -624.280720 & -7.25 \\
\hline 2 & TS3Ac & -624.261794 & -624.274391 & -7.90 \\
\hline 3 & TS3Go & -624.258565 & -624.270879 & -7.73 \\
\hline 4 & TS3Ao & -624.251265 & -624.277486 & -16.45 \\
\hline 5 & TS4Gc & -624.257779 & -624.268510 & -6.73 \\
\hline 6 & TS4Ac & -624.261671 & -624.274261 & -7.90 \\
\hline 7 & TS4Go & -624.258517 & -624.270999 & -7.83 \\
\hline 8 & TS4Ao & -624.249283 & -624.275746 & -16.61 \\
\hline 9 & $7 \mathbf{a}$ & -624.325897 & -624.338764 & -8.07 \\
\hline 10 & $7 b$ & -624.299700 & -624.308870 & -5.75 \\
\hline 11 & 1 & -431.172940 & -431.201137 & -17.69 \\
\hline 12 & 2 & -193.118131 & -193.122147 & -2.52 \\
\hline 13 & 3a & -624.368881 & -624.381360 & -7.83 \\
\hline 14 & $3 b$ & -624.340459 & -624.361807 & -13.40 \\
\hline 15 & $3 c$ & -624.367495 & -624.380279 & -8.02 \\
\hline 16 & 3d & -624.291290 & -624.313129 & -13.70 \\
\hline 17 & $4 \mathbf{a}$ & -624.362428 & -624.375798 & -8.39 \\
\hline 18 & $4 b$ & -624.344922 & -624.365863 & -13.14 \\
\hline 19 & $4 c$ & -624.298762 & -624.317067 & -11.49 \\
\hline 20 & TS' & -624.292496 & -624.308320 & -9.93 \\
\hline 21 & TS', & -624.297152 & -624.307661 & -6.59 \\
\hline 22 & I & -624.299555 & -624.309432 & -6.20 \\
\hline
\end{tabular}

General. All reactions were run under $\mathrm{N}_{2}$. Toluene was distilled under $\mathrm{N}_{2}$ from sodium/benzophenone ketyl. ${ }^{1} \mathrm{H}$ and ${ }^{13} \mathrm{C}$ NMR spectra were measured in $\mathrm{CDCl}_{3}$ with $\mathrm{Me}_{4} \mathrm{Si}$ as the internal standard. Pure $\gamma$-hydroxy ketones $(\gamma$-HKs) 3 and 4 were prepared following the previously described procedure. ${ }^{3 c}$

Addition Reaction of Lithium Enolates of Propiophenone to Propene Oxide. A 1.0 M lithium hexamethyldisilazane (LHMDS) solution in hexane $(12.0 \mathrm{~mL})$ was treated under stirring at $-50^{\circ} \mathrm{C}$ with a 
solution of the Propiophenone $(10.0 \mathrm{mmol})$ in anhydrous toluene $(4.0 \mathrm{~mL})$, added over a period of about $10 \mathrm{~min}$. After $1 \mathrm{~h}$ at the same temperature, a solution of the epoxide $(5.0 \mathrm{mmol})$ in anhydrous toluene $(4.0 \mathrm{~mL})$ was added over $1 \mathrm{~min}$. The reaction mixture was stirred at rt. At different times (see Table 1), aliquots $(1.0 \mathrm{~mL})$ of the reaction mixture were diluted with $\mathrm{Et}_{2} \mathrm{O}$ and treated with saturated aqueous $\mathrm{NH}_{4} \mathrm{Cl}$. Evaporation of the washed $(5 \%$ aqueous $\mathrm{HCl}$, and brine) organic solution afforded a crude reaction product $(0.10 \mathrm{~g})$ which was dissolved in anhydrous pyridine $(2.0 \mathrm{~mL})$ and treated with $\mathrm{Ac}_{2} \mathrm{O}$ $(1.0 \mathrm{~mL})$. After $18 \mathrm{~h}$ at $\mathrm{rt}$, dilution with toluene and evaporation of the organic solution (this operation was repeated three times) afforded a crude liquid product which was filtered through a short silical gel column. Elution with a 1:1 hexane/Et ${ }_{2} \mathrm{O}$ afforded a liquid product which was analyzed by ${ }^{1} \mathrm{H}$ NMR (see Table 1).

\section{Structure: TS3Gc}

Z-matrix (molden format)

zmat angstroms

c

c $1 \mathrm{cc} 2$

c $2 \operatorname{cc} 3 \quad 1 \operatorname{ccc} 3$

c $3 \operatorname{cc} 4 \quad 2 \operatorname{ccc} 4 \quad 1 \operatorname{dih} 4$

c $4 \operatorname{cc} 5 \quad 3 \operatorname{ccc} 5 \quad 2 \operatorname{dih} 5$

c $5 \operatorname{cc} 6 \quad 4 \operatorname{ccc} 6 \quad 3 \operatorname{dih} 6$

c $3 \operatorname{cc} 7 \quad 2 \operatorname{ccc} 7 \quad 1 \operatorname{dih} 7$

c $7 \operatorname{cc} 8 \quad 3 \operatorname{ccc} 8 \quad 2 \operatorname{dih} 8$

c $8 \operatorname{cc} 9 \quad 7 \operatorname{ccc} 9 \quad 3$ dih 9

o 7 oc10 3 occ10 2 dih10

li 10 lio11 7 lioc11 3 dih11 


$\begin{array}{lll}\text { o } 11 \text { oli12 } & 10 \text { olio12 } & 7 \text { dih12 } \\ \text { c } 12 \text { co13 } & 11 \text { coli13 } & 10 \text { dih13 } \\ \text { c } 13 \text { cc14 } & 12 \text { cco14 } & 11 \text { dih14 } \\ \text { c } 13 \text { cc15 } & 12 \text { cco15 } & 11 \text { dih15 } \\ \text { h } 4 \text { hc16 } & 3 \text { hcc16 } & 2 \text { dih16 } \\ \text { h } 5 \text { hc17 } & 4 \text { hcc17 } & 3 \text { dih17 } \\ \text { h } 6 \text { hc18 } & 5 \text { hcc18 } & 4 \text { dih18 } \\ \text { h } 1 \text { hc19 } & 2 \text { hcc19 } & 3 \text { dih19 } \\ \text { h } 2 \text { hc20 } & 3 \text { hcc20 } & 4 \text { dih20 } \\ \text { h } 8 \text { hc21 } & 7 \text { hcc21 } & 3 \text { dih21 } \\ \text { h } 9 \text { hc22 } & 8 \text { hcc22 } & 7 \text { dih22 } \\ \text { h } 9 \text { hc23 } & 8 \text { hcc23 } & 7 \text { dih23 } \\ \text { h } 9 \text { hc24 } & 8 \text { hcc24 } & 7 \text { dih24 } \\ \text { h } 15 \text { hc25 } & 13 \text { hcc25 } & 12 \text { dih25 } \\ \text { h } 13 \text { hc26 } & 12 \text { hco26 } & 11 \text { dih26 } \\ \text { h } 14 \text { hc27 } & 13 \text { hcc27 } & 15 \operatorname{dih} 27 \\ \text { h } 14 \text { hc28 } & 13 \text { hcc28 } & 15 \operatorname{dih} 28 \\ \text { h } 14 \text { hc29 } & 13 \text { hcc29 } & 15 \operatorname{dih} 29 \\ \text { h } 15 \text { hc30 } & 13 \text { hcc30 } & 12 \operatorname{dih} 30 \\ \text { variables } & & \end{array}$

cc2 1.395823

cc3 1.405998

ccc3 121.270

cc4 1.408029

ccc4 117.838

dih4 1.333

cc5 1.394913

ccc5 121.021

dih5 -1.278

cc6 1.398779

ccc6 120.344

dih6 0.395

cc7 $\quad 1.505698$

ccc7 119.012

dih7 179.889

cc8 1.401959 


\begin{tabular}{ll} 
ccc8 & 120.017 \\
dih8 & 161.852 \\
cc9 & 1.507402 \\
ccc9 & 123.521 \\
dih9 & 158.638 \\
oc10 & 1.296273 \\
occ10 & 116.589 \\
dih10 & -12.052 \\
lio11 & 1.818968 \\
lioc11 & 127.862 \\
dih11 & 97.327 \\
oli12 & 1.880205 \\
olio12 & 129.975 \\
dih12 & 26.820 \\
co13 & 1.408961 \\
coli13 & 91.962 \\
dih13 & 30.595 \\
cc14 & 1.524238 \\
cco14 & 114.131 \\
dih14 & 161.746 \\
cc15 & 1.483338 \\
cco15 & 88.268 \\
dih15 & -81.103 \\
hc16 & 1.085245 \\
hcc16 & 120.124 \\
dih16 & 177.328 \\
hc17 & 1.087278 \\
hcc17 & 119.589 \\
dih17 & 179.725 \\
hc18 & 1.087092 \\
hcc18 & 120.267 \\
dih18 & 179.807 \\
hc19 & 1.087372 \\
hcc19 & 119.755 \\
dih19 & 179.813 \\
hc20 & 1.085027 \\
& \\
\hline
\end{tabular}




\begin{tabular}{lc} 
hcc20 & 118.654 \\
dih20 & -177.565 \\
hc21 & 1.086046 \\
hcc21 & 117.408 \\
dih21 & 1.445 \\
hc22 & 1.100924 \\
hcc22 & 109.731 \\
dih22 & -106.722 \\
hc23 & 1.097677 \\
hcc23 & 113.260 \\
dih23 & 134.633 \\
hc24 & 1.093742 \\
hcc24 & 111.465 \\
dih24 & 11.844 \\
hc25 & 1.071979 \\
hcc25 & 121.096 \\
dih25 & 53.214 \\
hc26 & 1.098658 \\
hco26 & 113.805 \\
dih26 & 35.114 \\
hc27 & 1.095261 \\
hcc27 & 109.526 \\
dih27 & -167.235 \\
hc28 & 1.097485 \\
hcc28 & 111.100 \\
dih28 & 72.894 \\
hc29 & 1.095831 \\
hcc29 & 110.401 \\
dih29 & -48.318 \\
hc30 & 1.091170 \\
hcc30 & 117.868 \\
dih30 & -97.508 \\
constants \\
end & \\
& \\
\hline
\end{tabular}

Number of imaginary frequency: 1 (eigenvalue $=-456.2318)$ 
Thermochemical Data

Vacuum B3LYP Data

Electronic Energy=

-624.269160 Hartree/Particle

Zero-point correction=

$0.242374 \quad$ Hartree/Particle

Thermal correction to Energy=

0.257615. Hartree/Particle

Thermal correction to Enthalpy=

$0.258559 \quad$ Hartree/Particle

Thermal correction to Gibbs Free Energy=

$0.199620 \quad$ Hartree/Particle

Sum of electronic and zero-point Energies=

$-624.026786$

Hartree/Particle

Sum of electronic and thermal Energies=

$-624.011545$

Hartree/Particle

Sum of electronic and thermal Enthalpies=

$-624.010601$

Hartree/Particle

Sum of electronic and thermal Free Energies=

$-624.069540$

Hartree/Particle

Thermal translational Energy=

0.889

$\mathrm{kcal} / \mathrm{mol}$

Thermal rotational Energy=

0.889

$\mathrm{kcal} / \mathrm{mol}$

Translational Entropy=

41.756

$\mathrm{Cal} / \mathrm{mol}$

Rotational Entropy=

32.189

$\mathrm{Cal} / \mathrm{mol}$

Corrected Free Energy=

$-624.037240$

Hartree/Particle

Vacuum MP2 data

Electronic Energy=

$-622.220237$

Hartree/Particle

IEF-PCM Data $(\varepsilon=2.104)$

$<$ Psi(f)|H+V(f)/2|Psi(f) $>=$

$-624.280720 \quad$ Hartree/Particle

\section{Structure:TS3Ac}

Z-matrix (molden format)

zmat angstroms

$\begin{array}{lll}\text { c } & & \\ \text { c } 1 \operatorname{cc} 2 & & \\ \text { c } 2 \operatorname{cc} 3 & 1 \operatorname{cc} 3 & \\ \text { c } 3 \operatorname{cc} 4 & 2 \operatorname{ccc} 4 & 1 \operatorname{dih} 4 \\ \text { c } 4 \operatorname{cc} 5 & 3 \operatorname{cc} 5 & 2 \operatorname{dih} 5 \\ \text { c } 5 \operatorname{cc} 6 & 4 \operatorname{ccc} 6 & 3 \operatorname{dih} 6 \\ \text { c } 3 \operatorname{cc} 7 & 2 \operatorname{ccc} 7 & 1 \operatorname{dih} 7\end{array}$




\begin{tabular}{|c|c|c|c|}
\hline \multicolumn{2}{|c|}{ c $7 \mathrm{cc} 8$} & $3 \operatorname{ccc} 8$ & $2 \operatorname{dih} 8$ \\
\hline \multicolumn{2}{|c|}{ c $8 \mathrm{cc} 9$} & $7 \operatorname{ccc} 9$ & 3 dih9 \\
\hline \multicolumn{2}{|c|}{ o 7 oc10 } & 3 occ 10 & $2 \operatorname{dih} 10$ \\
\hline \multicolumn{2}{|c|}{ li 10 lio1 1} & 7 lioc11 & 3 dih1 1 \\
\hline \multicolumn{2}{|c|}{ o 11 oli12 } & 10 olio12 & $7 \operatorname{dih} 12$ \\
\hline \multicolumn{2}{|c|}{ c 12 co 13} & 11 coli 13 & $10 \operatorname{dih} 13$ \\
\hline \multicolumn{2}{|c|}{ c $13 \mathrm{cc} 14$} & 12 cco14 & $11 \operatorname{dih} 14$ \\
\hline \multicolumn{2}{|c|}{ c $13 \mathrm{cc} 15$} & 12 cco 15 & $11 \operatorname{dih} 15$ \\
\hline \multicolumn{2}{|c|}{ h 8 hc16 } & 7 hcc16 & $3 \operatorname{dih} 16$ \\
\hline \multicolumn{2}{|c|}{ h 14 hc17 } & 13 hcc17 & $12 \operatorname{dih} 17$ \\
\hline \multicolumn{2}{|c|}{ h 14 hc18 } & 13 hcc18 & $12 \operatorname{dih} 18$ \\
\hline \multicolumn{2}{|c|}{ h 13 hc19 } & 12 hco19 & $11 \operatorname{dih} 19$ \\
\hline \multicolumn{2}{|c|}{ h 15 hc 20} & 13 hcc 20 & $12 \operatorname{dih} 20$ \\
\hline \multicolumn{2}{|c|}{ h 15 hc 21} & 13 hcc 21 & $12 \operatorname{dih} 21$ \\
\hline \multicolumn{2}{|c|}{ h 15 hc22 } & 13 hсc 22 & $12 \operatorname{dih} 22$ \\
\hline \multicolumn{2}{|c|}{ h 9 hc23 } & 8 hcc 23 & $7 \operatorname{dih} 23$ \\
\hline \multicolumn{2}{|c|}{ h 9 hc24 } & 8 hcc 24 & $7 \operatorname{dih} 24$ \\
\hline \multicolumn{2}{|c|}{ h 9 hc 25} & 8 hcc 25 & $7 \operatorname{dih} 25$ \\
\hline \multicolumn{2}{|c|}{ h 4 hc26 } & 3 hcc 26 & $2 \operatorname{dih} 26$ \\
\hline $\mathrm{h}$ & $5 \mathrm{hc} 27$ & 4 hcc27 & $3 \operatorname{dih} 27$ \\
\hline $\mathrm{h}$ & $6 \mathrm{hc} 28$ & 5 hcc 28 & $4 \operatorname{dih} 28$ \\
\hline $\mathrm{h}$ & $1 \mathrm{hc} 29$ & 2 hcc 29 & $3 \operatorname{dih} 29$ \\
\hline & \multirow{2}{*}{$\begin{array}{l}2 \mathrm{hc} 30 \\
\text { ariables }\end{array}$} & 3 hcc30 & $4 \operatorname{dih} 30$ \\
\hline & & & \\
\hline \multicolumn{2}{|c|}{$\mathrm{cc} 2$} & 95434 & \\
\hline \multirow{2}{*}{$\begin{array}{l}\mathrm{cc} 3 \\
\mathrm{ccc}\end{array}$} & & 07472 & \\
\hline & 120 & 0.815 & \\
\hline $\mathrm{cc}^{2}$ & 1.4 & 06986 & \\
\hline $\begin{array}{l}\operatorname{cc} 4 \\
\operatorname{ccc}\end{array}$ & 118 & 8.196 & \\
\hline dih & -0 . & 382 & \\
\hline $\mathrm{cc}$ & 1.35 & 95124 & \\
\hline & 121 & 1.005 & \\
\hline dil & -0 . & 350 & \\
\hline $\operatorname{cct}$ & 1.3 & 97836 & \\
\hline & 120 & 0.166 & \\
\hline & & 692 & \\
\hline
\end{tabular}




$\begin{array}{lc}\text { cc7 } & 1.502519 \\ \text { ccc7 } & 123.825 \\ \text { dih7 } & -179.927 \\ \text { cc8 } & 1.426209 \\ \text { ccc8 } & 120.945 \\ \text { dih8 } & 9.020 \\ \text { cc9 } & 1.513523 \\ \text { ccc9 } & 121.709 \\ \text { dih9 } & 153.289 \\ \text { oc10 } & 1.284003 \\ \text { occ10 } & 116.937 \\ \text { dih10 } & -162.269 \\ \text { lio11 } & 2.034936 \\ \text { lioc11 } & 118.504 \\ \text { dih11 } & 97.891 \\ \text { oli12 } & 1.912197 \\ \text { olio12 } & 135.459 \\ \text { dih12 } & 29.453 \\ \text { co13 } & 1.400951 \\ \text { coli13 } & 117.338 \\ \text { dih13 } & 0.153 \\ \text { cc14 } & 1.513755 \\ \text { cco14 } & 86.310 \\ \text { dih14 } & -6.918 \\ \text { cc15 } & 1.519925 \\ \text { cco15 } & 114.898 \\ \text { dih15 } & -125.483 \\ \text { hc16 } & 1.086534 \\ \text { hcc16 } & 116.714 \\ \text { dih16 } & 5.287 \\ \text { hc17 } & 1.073628 \\ \text { hcc17 } & 110.158 \\ \text { dih17 } & 65.230 \\ \text { hc18 } & 1.081913 \\ \text { hcc18 } & 110.913 \\ \text { dih18 } & -70.765 \\ & \end{array}$




\begin{tabular}{ll} 
hc19 & 1.100344 \\
hco19 & 112.568 \\
dih19 & 106.192 \\
hc20 & 1.096524 \\
hcc20 & 110.583 \\
dih20 & 53.325 \\
hc21 & 1.095820 \\
hcc21 & 109.826 \\
dih21 & -65.799 \\
hc22 & 1.097776 \\
hcc22 & 110.963 \\
dih22 & 174.241 \\
hc23 & 1.099884 \\
hcc23 & 110.434 \\
dih23 & -88.341 \\
hc24 & 1.095244 \\
hcc24 & 111.832 \\
dih24 & 30.526 \\
hc25 & 1.096228 \\
hcc25 & 111.743 \\
dih25 & 152.391 \\
hc26 & 1.085133 \\
hcc26 & 118.403 \\
dih26 & -179.568 \\
hc27 & 1.087283 \\
hcc27 & 119.750 \\
dih27 & -179.252 \\
hc28 & 1.087071 \\
hcc28 & 120.310 \\
dih28 & 179.732 \\
hc29 & 1.087132 \\
hcc29 & 119.589 \\
dih29 & -179.240 \\
hc30 & 1.085131 \\
hcc30 & 120.473 \\
dih30 & -179.354 \\
& \\
\hline
\end{tabular}


constants

end

Number of imaginary frequency: 1 (eigenvalue $=-404.9881)$

Thermochemical Data

Vacuum B3LYP Data

Electronic Energy=

-624.261794 Hartree/Particle

Zero-point correction $=$

$0.243772 \quad$ Hartree/Particle

Thermal correction to Energy=

$0.258632 \quad$ Hartree/Particle

Thermal correction to Enthalpy=

$0.259576 \quad$ Hartree/Particle

Thermal correction to Gibbs Free Energy=

$0.201406 \quad$ Hartree/Particle

Sum of electronic and zero-point Energies=

-624.018022 Hartree/Particle

Sum of electronic and thermal Energies=

-624.003162 Hartree/Particle

Sum of electronic and thermal Enthalpies=

-624.002218 Hartree/Particle

Sum of electronic and thermal Free Energies=

$-624.060387$

Hartree/Particle

Thermal translational Energy=

0.889

$\mathrm{kcal} / \mathrm{mol}$

Thermal rotational Energy=

0.889

$\mathrm{kcal} / \mathrm{mol}$

Translational Entropy=

$41.756 \quad \mathrm{Cal} / \mathrm{mol}$

Rotational Entropy=

32.355

$\mathrm{Cal} / \mathrm{mol}$

Corrected Free Energy=

-624.028008 Hartree/Particle

Vacuum MP2 data

Electronic Energy=

$-622.218 .468$

Hartree/Particle

IEF-PCM Data $(\varepsilon=2.104)$

$<$ Psi(f) $|\mathrm{H}+\mathrm{V}(\mathrm{f}) / 2| \mathrm{Psi}(\mathrm{f})>=$

-624.274391 Hartree/Particle

\section{Structure: TS3Go}

Z-matrix (molden format)

zmat angstroms

c

c $1 \mathrm{cc} 2$

c $2 \operatorname{cc} 3 \quad 1 \operatorname{ccc} 3$ 


\begin{tabular}{|c|c|c|}
\hline c $3 \mathrm{cc} 4$ & $2 \operatorname{ccc} 4$ & $1 \operatorname{dih} 4$ \\
\hline c $4 \operatorname{cc} 5$ & $3 \operatorname{ccc} 5$ & $2 \operatorname{dih} 5$ \\
\hline $5 \operatorname{cc} 6$ & $4 \operatorname{ccc} 6$ & $3 \operatorname{dih} 6$ \\
\hline $3 \mathrm{cc} 7$ & $2 \operatorname{ccc} 7$ & $1 \operatorname{dih} 7$ \\
\hline $7 \operatorname{cc} 8$ & $3 \operatorname{ccc} 8$ & $2 \operatorname{dih} 8$ \\
\hline c $8 \operatorname{cc} 9$ & $7 \operatorname{ccc} 9$ & $3 \operatorname{dih} 9$ \\
\hline o 7 oc10 & 3 occ 10 & $2 \operatorname{dih} 10$ \\
\hline li 10 lio11 & 7 lioc11 & $3 \operatorname{dih} 11$ \\
\hline c 11 cli 12 & 10 clio12 & $7 \operatorname{dih} 12$ \\
\hline c $12 \mathrm{cc} 13$ & 11 ccli13 & $10 \operatorname{dih} 13$ \\
\hline c $12 \mathrm{cc} 14$ & 11 ccli14 & $10 \operatorname{dih} 14$ \\
\hline o 12 oc15 & 11 ocli15 & $10 \operatorname{dih} 15$ \\
\hline h 8 hc16 & 7 hcc16 & $3 \operatorname{dih} 16$ \\
\hline h 14 hc17 & 12 hcc17 & $15 \operatorname{dih} 17$ \\
\hline h 14 hc18 & 12 hcc 18 & $15 \operatorname{dih} 18$ \\
\hline h 12 hc19 & 11 hcli19 & $10 \operatorname{dih} 19$ \\
\hline h 13 hc20 & 12 hcc 20 & $14 \operatorname{dih} 20$ \\
\hline h 13 hc 21 & 12 hcc 21 & $14 \operatorname{dih} 21$ \\
\hline h 13 hc22 & 12 hcc 22 & $14 \operatorname{dih} 22$ \\
\hline h 9 hc 23 & 8 hcc 23 & $7 \operatorname{dih} 23$ \\
\hline h 9 hc 24 & 8 hcc 24 & $7 \operatorname{dih} 24$ \\
\hline h 9 hc 25 & 8 hcc 25 & $7 \operatorname{dih} 25$ \\
\hline h 4 hc 26 & 3 hcc 26 & $2 \operatorname{dih} 26$ \\
\hline h $5 \mathrm{hc} 27$ & 4 hcc27 & $3 \operatorname{dih} 27$ \\
\hline h 6 hc 28 & 5 hcc 28 & $4 \operatorname{dih} 28$ \\
\hline h 1 hc 29 & 2 hcc 29 & $3 \operatorname{dih} 29$ \\
\hline 2 hc30 & 3 hcc30 & $4 \operatorname{dih} 30$ \\
\hline
\end{tabular}

$\begin{array}{ll}\text { cc2 } & 1.395505 \\ \text { cc3 } & 1.406469 \\ \text { ccc3 } & 120.679 \\ \text { cc4 } & 1.406339 \\ \text { ccc4 } & 118.553 \\ \text { dih4 } & -0.836 \\ \text { cc5 } & 1.394362 \\ \text { ccc5 } & 120.763\end{array}$




\begin{tabular}{ll} 
dih5 & 0.847 \\
cc6 & 1.398537 \\
cc6 & 120.143 \\
dih6 & -0.276 \\
cc7 & 1.496530 \\
ccc7 & 123.001 \\
dih7 & -179.355 \\
cc8 & 1.421860 \\
ccc8 & 121.669 \\
dih8 & -19.408 \\
cc9 & 1.520689 \\
ccc9 & 121.063 \\
dih9 & 152.645 \\
oc10 & 1.289273 \\
occ10 & 117.649 \\
dih10 & 167.265 \\
lio11 & 1.789223 \\
lioc11 & 90.677 \\
dih11 & 134.720 \\
cli12 & 2.204494 \\
clio12 & 145.177 \\
dih12 & -47.835 \\
cc13 & 1.539292 \\
ccli13 & 82.279 \\
dih13 & 171.841 \\
cc14 & 1.513685 \\
ccli14 & 75.838 \\
dih14 & 49.940 \\
oc15 & 1.349973 \\
ocli15 & 158.977 \\
dih15 & 23.579 \\
hc16 & 1.085604 \\
hcc16 & 116.653 \\
dih16 & 6.817 \\
hcc17 & 1.081532 \\
& 115.520 \\
\hline
\end{tabular}




\begin{tabular}{|c|c|}
\hline $\operatorname{dih} 17$ & 69.928 \\
\hline hc18 & 1.082509 \\
\hline hcc18 & 114.603 \\
\hline $\operatorname{dih} 18$ & -71.105 \\
\hline hc19 & 1.123859 \\
\hline hcli19 & 66.953 \\
\hline $\operatorname{dih} 19$ & -73.086 \\
\hline hc20 & 1.094231 \\
\hline hec20 & 110.044 \\
\hline $\operatorname{dih} 20$ & -44.452 \\
\hline hc21 & 1.094925 \\
\hline hcc21 & 108.270 \\
\hline $\operatorname{dih} 21$ & -163.011 \\
\hline hc22 & 1.107543 \\
\hline hcc22 & 115.286 \\
\hline $\operatorname{dih} 22$ & 77.711 \\
\hline hc23 & 1.099691 \\
\hline hec23 & 110.768 \\
\hline $\operatorname{dih} 23$ & -86.605 \\
\hline hc24 & 1.099328 \\
\hline hec24 & 113.527 \\
\hline $\operatorname{dih} 24$ & 31.431 \\
\hline hc25 & 1.096560 \\
\hline hec 25 & 111.999 \\
\hline $\operatorname{dih} 25$ & 153.867 \\
\hline hc26 & 1.085007 \\
\hline hcc26 & 118.419 \\
\hline $\operatorname{dih} 26$ & -178.818 \\
\hline hc27 & 1.087093 \\
\hline hec27 & 119.791 \\
\hline $\operatorname{dih} 27$ & 179.773 \\
\hline hc28 & 1.087072 \\
\hline hec28 & 120.215 \\
\hline $\operatorname{dih} 28$ & -179.907 \\
\hline hc29 & 1.086975 \\
\hline hcc29 & 119.700 \\
\hline
\end{tabular}


$\operatorname{dih} 29 \quad 179.771$

hc30 1.085529

hcc30 120.373

dih30 $\quad 177.849$

constants

end

Number of imaginary frequency: 1 (eigenvalue $=-453.8215)$

Thermochemical Data

Vacuum B3LYP Data

Electronic Energy=

Zero-point correction $=$

Thermal correction to Energy=

Thermal correction to Enthalpy=

Thermal correction to Gibbs Free Energy=

Sum of electronic and zero-point Energies=

Sum of electronic and thermal Energies=

Sum of electronic and thermal Enthalpies=

Sum of electronic and thermal Free Energies=

Thermal translational Energy=

Thermal rotational Energy=

Translational Entropy=

Rotational Entropy=

Corrected Free Energy=

Vacuum MP2 data

Electronic Energy=

IEF-PCM Data $(\varepsilon=2.104)$

$<\operatorname{Psi}(\mathrm{f})|\mathrm{H}+\mathrm{V}(\mathrm{f}) / 2| \mathrm{Psi}(\mathrm{f})>=$
-624.258565 Hartree/Particle

$0.241642 \quad$ Hartree/Particle

$0.257102 \quad$ Hartree/Particle

$0.258046 \quad$ Hartree/Particle

$0.198365 \quad$ Hartree/Particle

-624.016956 Hartree/Particle

-624.001496 Hartree/Particle

$-624.000552 \quad$ Hartree/Particle

-624.060233 Hartree/Particle

$0.889 \mathrm{kcal} / \mathrm{mol}$

$0.889 \mathrm{kcal} / \mathrm{mol}$

$41.756 \mathrm{Cal} / \mathrm{mol}$

$32.288 \quad \mathrm{Cal} / \mathrm{mol}$

$-624.027886 \quad$ Hartree/Particle

$-622.212695 \quad$ Hartree/Particle

-624.270879 Hartree/Particle

\section{Strucuture: TS3Ao}

Z-matrix (molden format) 


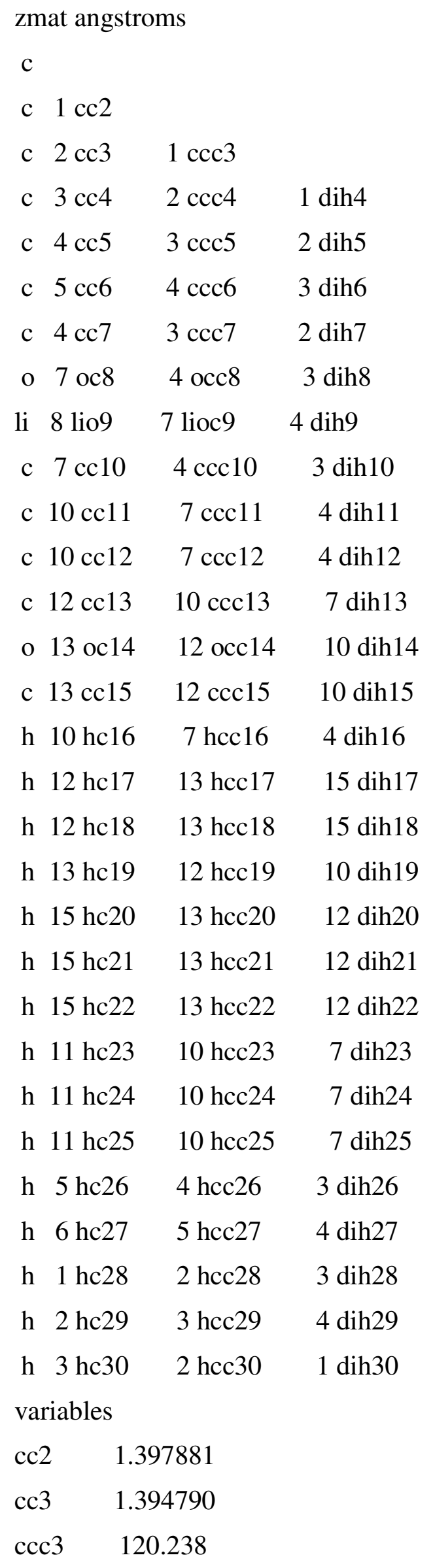




$\begin{array}{ll}\text { cc4 } & 1.406860 \\ \text { ccc4 } & 120.883 \\ \text { dih4 } & -0.064 \\ \text { cc5 } & 1.408180 \\ \text { ccc5 } & 118.292 \\ \text { dih5 } & 0.223 \\ \text { cc6 } & 1.394356 \\ \text { ccc6 } & 120.795 \\ \text { dih6 } & -0.280 \\ \text { cc7 } & 1.488455 \\ \text { ccc7 } & 118.675 \\ \text { dih7 } & 179.726 \\ \text { oc8 } & 1.300847 \\ \text { occ8 } & 117.312 \\ \text { dih8 } & -2.036 \\ \text { lio9 } & 1.722581 \\ \text { lioc9 } & 122.084 \\ \text { dih9 } & 179.135 \\ \text { cc10 } & 1.406966 \\ \text { ccc10 } & 122.804 \\ \text { dih10 } & 173.617 \\ \text { cc11 } & 1.514118 \\ \text { ccc11 } & 117.719 \\ \text { dih11 } & 166.045 \\ \text { cc12 } & 2.098907 \\ \text { ccc12 } & 100.328 \\ \text { dih12 } & -84.366 \\ \text { cc13 } & 1.497171 \\ \text { ccc13 } & 113.092 \\ \text { dih13 } & -177.492 \\ \text { oc14 } & 1.366773 \\ \text { occ14 } & 85.686 \\ \text { dih14 } & 175.112 \\ \text { cc15 } 15 & 1.529270 \\ \text { dih15 } & -67.788\end{array}$




$\begin{array}{ll}\text { hc16 } & 1.084206 \\ \text { hcc16 } & 118.755 \\ \text { dih16 } & 19.040 \\ \text { hc17 } & 1.084114 \\ \text { hcc17 } & 115.170 \\ \text { dih17 } & 44.895 \\ \text { hc18 } & 1.085924 \\ \text { hcc18 } & 116.095 \\ \text { dih18 } & -175.744 \\ \text { hc19 } & 1.110623 \\ \text { hcc19 } & 112.882 \\ \text { dih19 } & 60.282 \\ \text { hc20 } & 1.096538 \\ \text { hcc20 } & 109.210 \\ \text { dih20 } & -164.808 \\ \text { hc21 } & 1.100052 \\ \text { hcc21 } & 112.186 \\ \text { dih21 } & 75.375 \\ \text { hc22 } & 1.096710 \\ \text { hcc22 } & 110.326 \\ \text { dih22 } & -46.470 \\ \text { hc23 } & 1.092999 \\ \text { hcc23 } & 111.824 \\ \text { dih23 } & -167.712 \\ \text { hc24 } & 1.111653 \\ \text { hcc24 } & 112.713 \\ \text { dih24 } & 75.780 \\ \text { hc25 } & 1.108058 \\ \text { hcc25 } & 112.531 \\ \text { dih25 } & -48.267 \\ \text { hc26 } & 1.085283 \\ \text { hcc26 } & 120.451 \\ \text { dih26 } & 179.755 \\ \text { hc27 } & 1.087045 \\ \text { hcc27 } & 119.592 \\ \text { dih27 } & -179.824 \\ & \end{array}$


hc28 1.087044

hcc28 120.316

$\operatorname{dih} 28 \quad 179.904$

hc29 1.087300

hcc29 119.716

$\operatorname{dih} 29 \quad 179.798$

hc30 $\quad 1.084608$

hcc30 120.696

$\operatorname{dih} 30 \quad-179.849$

constants

end

Number of imaginary frequency: 1 (eigenvalue $=-527.0140$ )

Thermochemical Data 
Vacuum B3LYP Data

Electronic Energy=

Zero-point correction=

Thermal correction to Energy=

Thermal correction to Enthalpy=

Thermal correction to Gibbs Free Energy=

Sum of electronic and zero-point Energies=

Sum of electronic and thermal Energies=

Sum of electronic and thermal Enthalpies=

Sum of electronic and thermal Free Energies=

Thermal translational Energy=

Thermal rotational Energy=

Translational Entropy=

Rotational Entropy=

Corrected Free Energy=

Vacuum MP2 data

Electronic Energy=

IEF-PCM Data $(\varepsilon=2.104)$

$<\operatorname{Psi}(\mathrm{f})|\mathrm{H}+\mathrm{V}(\mathrm{f}) / 2| \mathrm{Psi}(\mathrm{f})>=$
-624.251265 Hartree/Particle

0.242019 Hartree/Particle

0.257297 Hartree/Particle

0.258241 Hartree/Particle

0.198374 Hartree/Particle

-624.009247 Hartree/Particle

-624.993968 Hartree/Particle

-624.993024 Hartree/Particle

Hartree/Particle

$0.889 \mathrm{kcal} / \mathrm{mol}$

$0.889 \mathrm{kcal} / \mathrm{mol}$

$41.756 \quad \mathrm{Cal} / \mathrm{mol}$

$32.406 \quad \mathrm{Cal} / \mathrm{mol}$

-624.020488 Hartree/Particle

-622.205330 Hartree/Particle

-624.277486 Hartree/Particle

\section{Structure: TS4Gc}

Z-matrix (molden format)

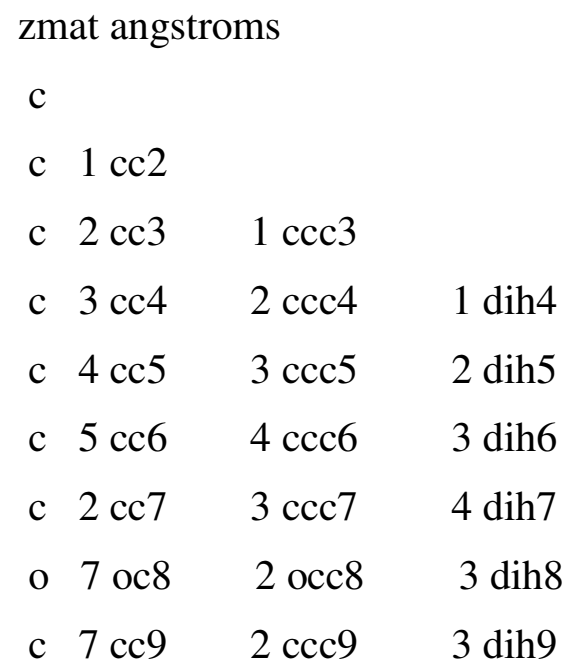




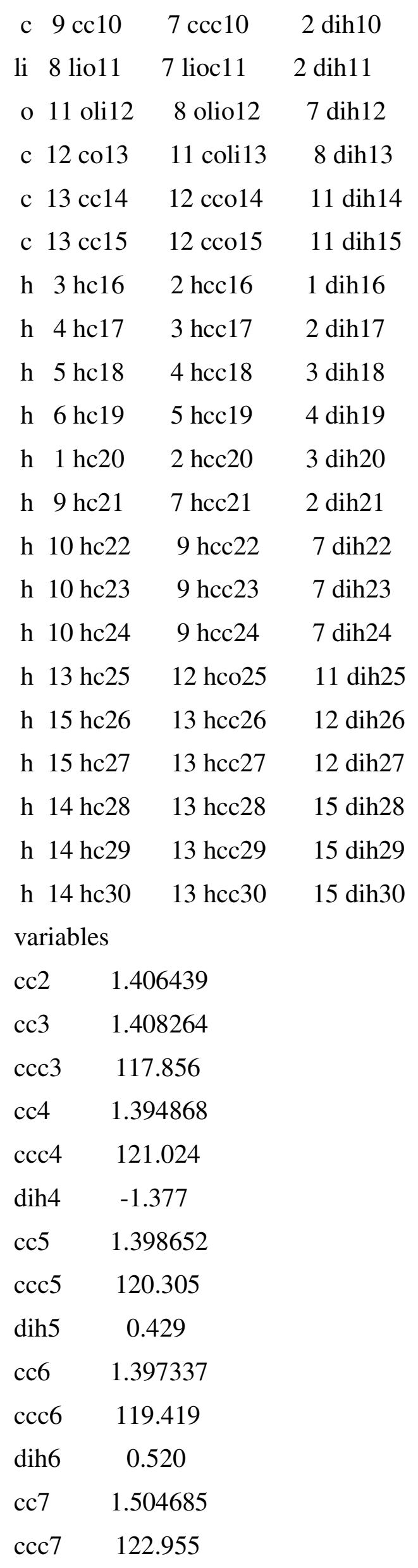




$\begin{array}{ll}\text { dih7 } & -179.883 \\ \text { oc8 } & 1.295607 \\ \text { occ8 } & 116.633 \\ \text { dih8 } & 165.084 \\ \text { cc9 } & 1.405118 \\ \text { ccc9 } & 119.702 \\ \text { dih9 } & -21.408 \\ \text { cc10 } & 1.505748 \\ \text { ccc10 } & 123.930 \\ \text { dih10 } & 155.050 \\ \text { lio11 } & 1.830825 \\ \text { lioc11 } & 127.037 \\ \text { dih11 } & 95.040 \\ \text { oli12 } & 1.893230 \\ \text { olio12 } & 134.565 \\ \text { dih12 } & 22.979 \\ \text { co13 } & 1.395599 \\ \text { coli13 } & 99.258 \\ \text { dih13 } & 35.958 \\ \text { cc14 } & 1.525473 \\ \text { cco14 } & 115.014 \\ \text { dih14 } & 57.984 \\ \text { cc15 } & 1.506810 \\ \text { cco15 } & 90.245 \\ \text { dih15 } & -70.268 \\ \text { hc16 } & 1.085255 \\ \text { hcc16 } & 120.053 \\ \text { dih16 } & 177.197 \\ \text { hc17 } & 1.087225 \\ \text { hcc17 } & 119.619 \\ \text { dih17 } & 179.752 \\ \text { hc18 } & 1.087098 \\ \text { hcc18 } & 120.259 \\ \text { dih18 } & 179.858 \\ \text { hc19 } & 1.087307 \\ \text { hcc19 } & 120.103\end{array}$




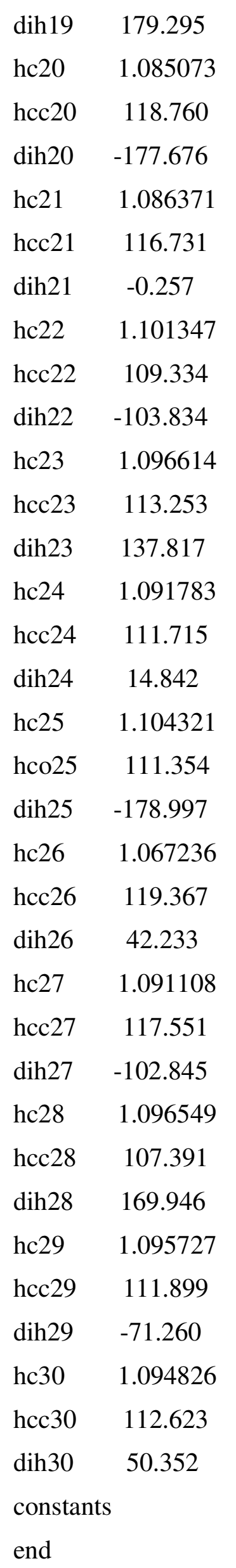


Number of imaginary frequency: 1 (eigenvalue $=-453.0426$ )

Thermochemical Data

Vacuum B3LYP Data

Electronic Energy=

Zero-point correction=

Thermal correction to Energy=

Thermal correction to Enthalpy=

Thermal correction to Gibbs Free Energy=

Sum of electronic and zero-point Energies=

Sum of electronic and thermal Energies=

Sum of electronic and thermal Enthalpies=

Sum of electronic and thermal Free Energies=

Thermal translational Energy=

Thermal rotational Energy=

Translational Entropy=

Rotational Entropy=

Corrected Free Energy=

Vacuum MP2 data

Electronic Energy=

IEF-PCM Data $(\varepsilon=2.104)$

$<\mathrm{Psi}(\mathrm{f})|\mathrm{H}+\mathrm{V}(\mathrm{f}) / 2| \mathrm{Psi}(\mathrm{f})>=$
-624.257779 Hartree/Particle

0.242486 Hartree/Particle

0.257616 Hartree/Particle

$0.258561 \quad$ Hartree/Particle

$0.199855 \quad$ Hartree/Particle

-624.015293 Hartree/Particle

-624.000162 Hartree/Particle

$-624.999218 \quad$ Hartree/Particle

Hartree/Particle

$\mathrm{kcal} / \mathrm{mol}$

$\mathrm{kcal} / \mathrm{mol}$

$\mathrm{Cal} / \mathrm{mol}$

$\mathrm{Cal} / \mathrm{mol}$

$-624.025638$

Hartree/Particle

$-622.268510 \quad$ Hartree/Particle

$-624.268510 \quad$ Hartree/Particle

\section{Structure: TS4Ac}

Z-matrix (molden format)

zmat angstroms

c

c $1 \mathrm{cc} 2$

c $2 \operatorname{cc} 3 \quad 1 \operatorname{ccc} 3$

c $3 \operatorname{cc} 4 \quad 2 \operatorname{ccc} 4 \quad 1 \operatorname{dih} 4$ 


\begin{tabular}{|c|c|c|}
\hline $4 \operatorname{cc} 5$ & $3 \operatorname{ccc} 5$ & $2 \operatorname{dih} 5$ \\
\hline 5 cc6 & $4 \operatorname{ccc} 6$ & 3 dih6 \\
\hline $5 \mathrm{cc} 7$ & $4 \operatorname{ccc} 7$ & $3 \operatorname{dih} 7$ \\
\hline $7 \operatorname{cc} 8$ & $5 \operatorname{ccc} 8$ & $4 \operatorname{dih} 8$ \\
\hline c $8 \operatorname{cc} 9$ & $7 \operatorname{ccc} 9$ & $5 \operatorname{dih} 9$ \\
\hline o 7 oc 10 & 5 occ 10 & $4 \operatorname{dih} 10$ \\
\hline i 10 lio11 & 7 lioc11 & $5 \operatorname{dih} 11$ \\
\hline o 11 oli12 & 10 olio12 & $7 \operatorname{dih} 12$ \\
\hline c 12 col3 & 11 coli13 & $10 \operatorname{dih} 13$ \\
\hline c $13 \mathrm{cc} 14$ & $12 \operatorname{cco} 14$ & $11 \operatorname{dih} 14$ \\
\hline c $13 \mathrm{cc} 15$ & $12 \operatorname{cco} 15$ & $11 \operatorname{dih} 15$ \\
\hline h 8 hc16 & 7 hcc16 & $5 \operatorname{dih} 16$ \\
\hline h 14 hc17 & 13 hcc17 & $12 \operatorname{dih} 17$ \\
\hline h 14 hc18 & 13 hcc18 & $12 \operatorname{dih} 18$ \\
\hline h 13 hc19 & 12 hco19 & 11 dih1s \\
\hline h 9 hc20 & 8 hcc20 & $7 \operatorname{dih} 20$ \\
\hline h $9 \mathrm{hc} 21$ & 8 hcc 21 & $7 \operatorname{dih} 21$ \\
\hline h 9 hc 22 & 8 hcc 22 & $7 \operatorname{dih} 22$ \\
\hline h 6 hc23 & 5 hcc 23 & $4 \operatorname{dih} 23$ \\
\hline h 1 hc 24 & 2 hсc24 & $3 \operatorname{dih} 24$ \\
\hline h 2 hc 25 & 3 hcc 25 & $4 \operatorname{dih} 25$ \\
\hline h $3 \mathrm{hc} 26$ & 2 hсc26 & $1 \operatorname{dih} 26$ \\
\hline h 4 hc27 & 3 hec27 & $2 \operatorname{dih} 27$ \\
\hline h 15 hc 28 & 13 hcc 28 & $12 \operatorname{dih} 28$ \\
\hline h 15 hc 29 & 13 hcc29 & $12 \operatorname{dih} 29$ \\
\hline h 15 hc30 & 13 hcc30 & $12 \operatorname{dih} 30$ \\
\hline
\end{tabular}

$\begin{array}{lc}\mathrm{cc} 2 & 1.398141 \\ \mathrm{cc} 3 & 1.398107 \\ \mathrm{ccc} 3 & 119.526 \\ \mathrm{cc} 4 & 1.395466 \\ \mathrm{ccc} 4 & 120.260 \\ \operatorname{dih} 4 & 0.527 \\ \mathrm{cc5} & 1.407128 \\ \operatorname{ccc5} & 120.880 \\ \operatorname{dih} 5 & 0.283\end{array}$




$\begin{array}{ll}\text { cc6 } & 1.406514 \\ \text { ccc6 } & 118.129 \\ \text { dih6 } & -1.238 \\ \text { cc7 } & 1.502490 \\ \text { ccc7 } & 122.841 \\ \text { dih7 } & -179.341 \\ \text { cc8 } & 1.427900 \\ \text { ccc8 } & 120.163 \\ \text { dih8 } & -21.928 \\ \text { cc9 } & 1.513405 \\ \text { ccc9 } & 122.048 \\ \text { dih9 } & 152.179 \\ \text { oc10 } & 1.283841 \\ \text { occ10 } & 117.393 \\ \text { dih10 } & 167.979 \\ \text { lio11 } & 2.019700 \\ \text { lioc11 } & 120.247 \\ \text { dih11 } & 99.603 \\ \text { oli12 } & 1.910448 \\ \text { olio12 } & 135.607 \\ \text { dih12 } & 26.461 \\ \text { co13 } & 1.400729 \\ \text { coli13 } & 117.264 \\ \text { dih13 } & 1.993 \\ \text { cc14 } & 1.513398 \\ \text { cco14 } & 86.279 \\ \text { dih14 } & -7.498 \\ \text { cc15 } & 1.520671 \\ \text { cco15 } & 114.983 \\ \text { dih15 } & 111.266 \\ \text { hc16 } & 1.086683 \\ \text { hcc16 } & 116.637 \\ \text { dih16 } & 2.406 \\ \text { hc17 } & 1.074388 \\ \text { hcc17 } & 109.705 \\ \text { dih17 } & 65.448 \\ & \end{array}$




$\begin{array}{ll}\text { hc18 } & 1.080540 \\ \text { hcc18 } & 111.770 \\ \text { dih18 } & -70.993 \\ \text { hc19 } & 1.099864 \\ \text { hco19 } & 112.374 \\ \text { dih19 } & -120.561 \\ \text { hc20 } & 1.100030 \\ \text { hcc20 } & 110.474 \\ \text { dih20 } & -89.268 \\ \text { hc21 } & 1.095471 \\ \text { hcc21 } & 111.786 \\ \text { dih21 } & 29.555 \\ \text { hc22 } & 1.096108 \\ \text { hcc22 } & 111.839 \\ \text { dih22 } & 151.361 \\ \text { hc23 } & 1.085188 \\ \text { hcc23 } & 118.630 \\ \text { dih23 } & -177.661 \\ \text { hc24 } & 1.087254 \\ \text { hcc24 } & 120.072 \\ \text { dih24 } & 179.309 \\ \text { hc25 } & 1.087086 \\ \text { hcc25 } & 120.200 \\ \text { dih25 } & 179.883 \\ \text { hc26 } & 1.087121 \\ \text { hcc26 } & 120.093 \\ \text { dih26 } & -178.942 \\ \text { hc27 } & 1.085317 \\ \text { hcc27 } & 118.957 \\ \text { dih27 } & -178.414 \\ \text { hc28 } & 1.095953 \\ \text { hcc28 } & 109.676 \\ \text { dih28 } & 65.046 \\ \text { hc29 } & 1.097784 \\ \text { hcc29 } & 111.040 \\ \text { dih29 } & -175.058 \\ & \end{array}$


hc30 1.096383

hec30 110.687

$\operatorname{dih} 30 \quad-54.047$

constants

end

Number of imaginary frequency: 1 (eigenvalue $=-409.1197)$

Thermochemical Data

\section{Vacuum B3LYP Data}

Electronic Energy=

Zero-point correction $=$

Thermal correction to Energy=

Thermal correction to Enthalpy=

Thermal correction to Gibbs Free Energy=

Sum of electronic and zero-point Energies=

Sum of electronic and thermal Energies $=$

Sum of electronic and thermal Enthalpies=

Sum of electronic and thermal Free Energies=

Thermal translational Energy=

Thermal rotational Energy=

Translational Entropy=

Rotational Entropy=

Corrected Free Energy=

Vacuum MP2 data

Electronic Energy=

IEF-PCM Data $(\varepsilon=2.104)$

$<\mathrm{Psi}(\mathrm{f})|\mathrm{H}+\mathrm{V}(\mathrm{f}) / 2| \mathrm{Psi}(\mathrm{f})>=$
$-622.218249 \quad$ Hartree/Particle

-624.261671 Hartree/Particle

$0.243826 \quad$ Hartree/Particle

0.258629 Hartree/Particle

0.259573 Hartree/Particle

$0.201744 \quad$ Hartree/Particle

-624.017846 Hartree/Particle

$-624.003043 \quad$ Hartree/Particle

-624.002099 Hartree/Particle

Hartree/Particle

$\mathrm{kcal} / \mathrm{mol}$

$\mathrm{kcal} / \mathrm{mol}$

$\mathrm{Cal} / \mathrm{mol}$

$\mathrm{Cal} / \mathrm{mol}$

-624.027571 Hartree/Particle

-624.274261 Hartree/Particle

Structure: TS4Go

Z-matrix (molden format) 


\begin{tabular}{|c|c|c|c|}
\hline \multicolumn{4}{|c|}{ mat angstroms } \\
\hline \multicolumn{4}{|c|}{$c$} \\
\hline \multicolumn{4}{|c|}{ c $1 \mathrm{cc} 2$} \\
\hline \multicolumn{2}{|c|}{ c $2 \operatorname{cc} 3$} & $1 \operatorname{ccc} 3$ & \\
\hline \multicolumn{2}{|c|}{ c $3 \mathrm{cc} 4$} & $2 \operatorname{ccc} 4$ & $1 \mathrm{dih} 4$ \\
\hline \multicolumn{2}{|c|}{ c $4 \operatorname{cc} 5$} & $3 \operatorname{ccc} 5$ & $2 \operatorname{dih} 5$ \\
\hline \multicolumn{2}{|c|}{ c 5 cc6 } & $4 \operatorname{ccc} 6$ & 3 dih6 \\
\hline \multicolumn{2}{|c|}{ c $3 \mathrm{cc} 7$} & $2 \operatorname{ccc} 7$ & $1 \operatorname{dih} 7$ \\
\hline \multicolumn{2}{|c|}{ o 7 oc8 } & 3 occ 8 & $2 \operatorname{dih} 8$ \\
\hline & $7 \mathrm{cc} 9$ & $3 \operatorname{ccc} 9$ & $2 \operatorname{dih} 9$ \\
\hline & $9 \operatorname{cc} 10$ & $7 \operatorname{ccc} 10$ & $3 \operatorname{dih} 10$ \\
\hline \multicolumn{2}{|c|}{ i 8 lio11 } & 7 lioc11 & $3 \operatorname{dih} 11$ \\
\hline \multicolumn{2}{|c|}{ c 11 cli 12} & 8 clio12 & $7 \operatorname{dih} 12$ \\
\hline \multicolumn{2}{|c|}{ o 12 oc 13} & 11 ocli13 & $8 \operatorname{dih} 13$ \\
\hline \multicolumn{2}{|c|}{ c 12 cc14 } & 11 ccli14 & $8 \operatorname{dih} 14$ \\
\hline \multicolumn{2}{|c|}{ c 12 cc15 } & 11 ccli 15 & $8 \operatorname{dih} 15$ \\
\hline \multicolumn{2}{|c|}{ h 9 hc16 } & 7 hcc16 & $3 \operatorname{dih} 16$ \\
\hline \multicolumn{2}{|c|}{ h 10 hc17 } & 9 hcc17 & $7 \operatorname{dih} 17$ \\
\hline \multicolumn{2}{|c|}{ h 10 hc18 } & 9 hcc18 & $7 \operatorname{dih} 18$ \\
\hline \multicolumn{2}{|c|}{ h 10 hc19 } & 9 hcc19 & $7 \operatorname{dih} 19$ \\
\hline \multicolumn{2}{|c|}{ h 4 hc20 } & 3 hcc20 & $2 \operatorname{dih} 20$ \\
\hline \multicolumn{2}{|c|}{ h 5 hc21 } & 4 hcc 21 & $3 \operatorname{dih} 21$ \\
\hline \multicolumn{2}{|c|}{ h 6 hc22 } & 5 hcc 22 & $4 \operatorname{dih} 22$ \\
\hline \multicolumn{2}{|c|}{ h 1 hc23 } & 2 hcc 23 & $3 \operatorname{dih} 23$ \\
\hline \multicolumn{2}{|c|}{ h 2 hc 24} & 3 hcc24 & $4 \operatorname{dih} 24$ \\
\hline \multicolumn{2}{|c|}{ h 14 hc 25} & 12 hcc 25 & $15 \operatorname{dih} 25$ \\
\hline \multicolumn{2}{|c|}{ h 14 hc26 } & 12 hcc 26 & $15 \operatorname{dih} 26$ \\
\hline \multicolumn{2}{|c|}{ h 12 hc 27} & 11 hcli27 & $8 \operatorname{dih} 27$ \\
\hline \multicolumn{2}{|c|}{ h 15 hc 28} & 12 hcc 28 & $14 \operatorname{dih} 28$ \\
\hline & 15 hc29 & 12 hcc 29 & $14 \operatorname{dih} 29$ \\
\hline & 15 hc30 & 12 hcc30 & $14 \operatorname{dih} 30$ \\
\hline & riables & & \\
\hline & 1.3 & 470 & \\
\hline & 1.4 & 6291 & \\
\hline & 12 & .797 & \\
\hline & 1.4 & 641 & \\
\hline
\end{tabular}




$\begin{array}{ll}\text { ccc4 } & 118.506 \\ \text { dih4 } & -0.698 \\ \text { cc5 } & 1.395350 \\ \text { ccc5 } & 120.696 \\ \text { dih5 } & 0.841 \\ \text { cc6 } & 1.398268 \\ \text { ccc6 } & 120.202 \\ \text { dih6 } & -0.381 \\ \text { cc7 } & 1.497025 \\ \text { ccc7 } & 118.271 \\ \text { dih7 } & -179.443 \\ \text { oc8 } & 1.288685 \\ \text { occ8 } & 117.692 \\ \text { dih8 } & 7.234 \\ \text { cc9 } & 1.421647 \\ \text { ccc9 } & 121.984 \\ \text { dih9 } & -166.548 \\ \text { cc10 } & 1.521574 \\ \text { ccc10 } & 120.628 \\ \text { dih10 } & -152.875 \\ \text { lio11 } & 1.795766 \\ \text { lioc11 } & 90.230 \\ \text { dih11 } & -134.432 \\ \text { cli12 } & 2.205174 \\ \text { clio12 } & 140.519 \\ \text { dih12 } & 51.986 \\ \text { oc13 } & 1.348534 \\ \text { ocli13 } & 158.889 \\ \text { dih13 } & -95.001 \\ \text { cc14 } & 1.512294 \\ \text { ccli14 } & 76.364 \\ \text { dih14 } & -64.484 \\ \text { cc15 } & 1.537729 \\ \text { ccli15 } & 83.028 \\ \text { dih15 } & 56.756 \\ \text { hc16 } & 1.085519 \\ & \end{array}$




$\begin{array}{ll}\text { hcc16 } & 116.942 \\ \text { dih16 } & -7.737 \\ \text { hc17 } & 1.096712 \\ \text { hcc17 } & 111.924 \\ \text { dih17 } & -156.323 \\ \text { hc18 } & 1.099642 \\ \text { hcc18 } & 110.845 \\ \text { dih18 } & 84.226 \\ \text { hc19 } & 1.099998 \\ \text { hcc19 } & 113.778 \\ \text { dih19 } & -33.834 \\ \text { hc20 } & 1.085416 \\ \text { hcc20 } & 120.450 \\ \text { dih20 } & -178.077 \\ \text { hc21 } & 1.086992 \\ \text { hcc21 } & 119.675 \\ \text { dih21 } & -179.902 \\ \text { hc22 } & 1.087067 \\ \text { hcc22 } & 120.114 \\ \text { dih22 } & -179.815 \\ \text { hc23 } & 1.087125 \\ \text { hcc23 } & 119.781 \\ \text { dih23 } & -179.942 \\ \text { hc24 } & 1.084871 \\ \text { hcc24 } & 118.384 \\ \text { dih24 } & 178.933 \\ \text { hc25 } & 1.082695 \\ \text { hcc25 } & 114.590 \\ \text { dih25 } & 41.485 \\ \text { hc26 } & 1.081522 \\ \text { hcc26 } & 115.470 \\ \text { dih26 } & -177.561 \\ \text { hc27 } & 1.130007 \\ \text { hcli27 } & 65.517 \\ \text { dih27 } & 171.921 \\ \text { hc28 } & 1.094328 \\ & \end{array}$




$\begin{array}{ll}\text { hcc28 } & 109.640 \\ \text { dih28 } & -44.349 \\ \text { hc29 } & 1.095084 \\ \text { hcc29 } & 108.176 \\ \text { dih29 } & -162.535 \\ \text { hc30 } & 1.104742 \\ \text { hcc30 } & 115.163 \\ \text { dih30 } & 77.324 \\ \text { constants } \\ \text { end }\end{array}$

Number of imaginary frequency: 1 (eigenvalue $=-452.2615)$

Thermochemical Data

Vacuum B3LYP Data

Electronic Energy=

Zero-point correction=

Thermal correction to Energy=

Thermal correction to Enthalpy=

Thermal correction to Gibbs Free Energy=

Sum of electronic and zero-point Energies=

Sum of electronic and thermal Energies=

Sum of electronic and thermal Enthalpies=

Sum of electronic and thermal Free Energies=

Thermal translational Energy=

Thermal rotational Energy=

Translational Entropy=

Rotational Entropy=

Corrected Free Energy=

$$
\text { Vacuum MP2 data }
$$

Electronic Energy=

IEF-PCM Data $(\varepsilon=2.104)$
-624.258517 Hartree/Particle

0.241625 Hartree/Particle

0.257081 Hartree/Particle

0.258025 Hartree/Particle

0.198104 Hartree/Particle

-624.016892 Hartree/Particle

-624.001437 Hartree/Particle

-624.000492 Hartree/Particle

-624.060413 Hartree/Particle

$0.889 \mathrm{kcal} / \mathrm{mol}$

$0.889 \quad \mathrm{kcal} / \mathrm{mol}$

$41.756 \quad \mathrm{Cal} / \mathrm{mol}$

$32.237 \quad \mathrm{Cal} / \mathrm{mol}$

$-624.028090 \quad$ Hartree/Particle

-622.212817 Hartree/Particle

-624.270999 Hartree/Particle 
Structure:TS4Ao

Z-matrix (molden format)

zmat angstroms

c

c $1 \mathrm{cc} 2$

c $2 \operatorname{cc} 3 \quad 1 \operatorname{ccc} 3$

c $3 \operatorname{cc} 4 \quad 2 \operatorname{ccc} 4 \quad 1 \operatorname{dih} 4$

c $4 \operatorname{cc5} 3 \operatorname{ccc} 5 \quad 2 \operatorname{dih} 5$

c 5 cc6 4 cce $6 \quad 3$ dih 6

$\begin{array}{lll}\text { c } 3 \operatorname{cc} 7 & 2 \operatorname{ccc} 7 & 1 \operatorname{dih} 7\end{array}$

c $7 \operatorname{cc} 8 \quad 3 \operatorname{ccc} 8 \quad 2 \operatorname{dih} 8$

c $8 \operatorname{cc} 9 \quad 7 \operatorname{ccc} 9 \quad 3$ dih 9

o 7 oc10 3 occ10 2 dih10

li 10 lio11 7 lioc11 3 dih11

c $8 \operatorname{cc} 12 \quad 7 \operatorname{ccc} 12 \quad 3 \operatorname{dih} 12$

c $12 \operatorname{cc} 13 \quad 8 \operatorname{ccc} 13 \quad 7 \operatorname{dih} 13$

c 13 cc14 12 ccc14 8 dih14

o 13 oc15 12 occ15 8 dih15

h 8 hc16 7 hcc16 3 dih16

h 9 hc17 8 hcc17 7 dih17

h 9 hc18 8 hcc18 7 dih18

h 9 hc19 8 hcc19 7 dih19

h 14 hc20 13 hcc20 $12 \operatorname{dih} 20$

h 14 hc21 13 hcc $21 \quad 12 \operatorname{dih} 21$

h 14 hc22 13 hcc22 $12 \operatorname{dih} 22$

h 13 hc23 12 hcc23 $8 \operatorname{dih} 23$

h 12 hc24 13 hcc $24 \quad 15 \operatorname{dih} 24$

h 12 hc $25 \quad 13$ hec $25 \quad 15 \operatorname{dih} 25$

h 4 hc26 3 hcc26 2 dih26

h 5 hc27 4 hcc $27 \quad 3 \operatorname{dih} 27$

h 6 hc $28 \quad 5$ hcc $28 \quad 4$ dih 28

h 1 hc29 2 hcc29 $3 \operatorname{dih} 29$

h 2 hc30 3 hcc30 $4 \operatorname{dih} 30$ 


\begin{tabular}{|c|c|}
\hline \multicolumn{2}{|c|}{ variables } \\
\hline $\operatorname{cc} 2$ & 1.394504 \\
\hline $\operatorname{cc} 3$ & 1.408200 \\
\hline $\operatorname{ccc} 3$ & 120.731 \\
\hline $\operatorname{cc} 4$ & 1.406962 \\
\hline $\operatorname{ccc} 4$ & 118.369 \\
\hline $\operatorname{dih} 4$ & -0.113 \\
\hline $\operatorname{cc} 5$ & 1.394561 \\
\hline $\operatorname{ccc} 5$ & 120.849 \\
\hline $\operatorname{dih} 5$ & 0.411 \\
\hline $\operatorname{cc} 6$ & 1.398143 \\
\hline $\operatorname{ccc} 6$ & 120.215 \\
\hline dih6 & -0.331 \\
\hline $\operatorname{cc} 7$ & 1.487576 \\
\hline $\operatorname{ccc} 7$ & 122.935 \\
\hline $\operatorname{dih} 7$ & 179.483 \\
\hline $\operatorname{cc} 8$ & 1.409402 \\
\hline $\operatorname{ccc} 8$ & 122.624 \\
\hline $\operatorname{dih} 8$ & -2.941 \\
\hline $\operatorname{cc} 9$ & 1.514685 \\
\hline $\operatorname{ccc} 9$ & 117.020 \\
\hline dih9 & -165.212 \\
\hline oc 10 & 1.299931 \\
\hline occ10 & 117.321 \\
\hline $\operatorname{dih} 10$ & 174.159 \\
\hline lio11 & 1.722177 \\
\hline lioc11 & 122.223 \\
\hline $\operatorname{dih} 11$ & 179.645 \\
\hline $\operatorname{cc} 12$ & 2.099560 \\
\hline $\operatorname{ccc} 12$ & 101.195 \\
\hline $\operatorname{dih} 12$ & 82.055 \\
\hline $\mathrm{cc} 13$ & 1.500926 \\
\hline $\operatorname{ccc} 13$ & 115.371 \\
\hline $\operatorname{dih} 13$ & -161.915 \\
\hline $\operatorname{cc} 14$ & 1.530224 \\
\hline $\operatorname{ccc} 14$ & 118.648 \\
\hline
\end{tabular}




\begin{tabular}{|c|c|}
\hline $\operatorname{dih} 14$ & -59.482 \\
\hline oc15 & 1.364868 \\
\hline occ15 & 86.127 \\
\hline $\operatorname{dih} 15$ & -176.389 \\
\hline hc16 & 1.085170 \\
\hline hcc16 & 118.190 \\
\hline $\operatorname{dih} 16$ & -20.896 \\
\hline hc17 & 1.108424 \\
\hline hcc17 & 112.321 \\
\hline $\operatorname{dih} 17$ & 47.857 \\
\hline hc18 & 1.091886 \\
\hline hcc18 & 112.030 \\
\hline $\operatorname{dih} 18$ & 167.123 \\
\hline hc19 & 1.111352 \\
\hline hcc19 & 112.898 \\
\hline $\operatorname{dih} 19$ & -76.081 \\
\hline hc20 & 1.099351 \\
\hline hcc20 & 112.435 \\
\hline $\operatorname{dih} 20$ & 77.763 \\
\hline hc21 & 1.096514 \\
\hline hcc21 & 108.561 \\
\hline $\operatorname{dih} 21$ & -162.889 \\
\hline hc22 & 1.097251 \\
\hline hcc22 & 110.717 \\
\hline $\operatorname{dih} 22$ & -45.032 \\
\hline hc23 & 1.110942 \\
\hline hcc23 & 111.359 \\
\hline $\operatorname{dih} 23$ & 68.516 \\
\hline hc24 & 1.086917 \\
\hline hec24 & 115.537 \\
\hline $\operatorname{dih} 24$ & -66.621 \\
\hline hc25 & 1.082691 \\
\hline hcc 25 & 114.491 \\
\hline $\operatorname{dih} 25$ & 70.809 \\
\hline hc26 & 1.084638 \\
\hline $\operatorname{cc} 26$ & 118.432 \\
\hline
\end{tabular}




$\begin{array}{lc}\text { dih26 } & -179.574 \\ \text { hc27 } & 1.087298 \\ \text { hcc27 } & 119.735 \\ \text { dih27 } & 179.837 \\ \text { hc28 } & 1.087058 \\ \text { hcc28 } & 120.291 \\ \text { dih28 } & -179.743 \\ \text { hc29 } & 1.086980 \\ \text { hcc29 } & 119.585 \\ \text { dih29 } & 179.473 \\ \text { hc30 } & 1.085341 \\ \text { hcc30 } & 120.449 \\ \text { dih30 } & 178.950 \\ \text { constants } & \\ \text { end } & \end{array}$

Number of imaginary frequency: 1 (eigenvalue $=-515.1172)$

Thermochemical Data 
Vacuum B3LYP Data

Electronic Energy=

Zero-point correction=

Thermal correction to Energy=

Thermal correction to Enthalpy=

Thermal correction to Gibbs Free Energy=

Sum of electronic and zero-point Energies=

Sum of electronic and thermal Energies=

Sum of electronic and thermal Enthalpies=

Sum of electronic and thermal Free Energies=

Thermal translational Energy=

Thermal rotational Energy=

Translational Entropy=

Rotational Entropy=

Corrected Free Energy=

Vacuum MP2 data

Electronic Energy=

IEF-PCM Data $(\varepsilon=2.104)$

$<\operatorname{Psi}(\mathrm{f})|\mathrm{H}+\mathrm{V}(\mathrm{f}) / 2| \mathrm{Psi}(\mathrm{f})>=$
-624.249283 Hartree/Particle

0.242115 Hartree/Particle

0.257323 Hartree/Particle

0.258267 Hartree/Particle

0.198735 Hartree/Particle

-624.007168 Hartree/Particle

-623.991960 Hartree/Particle

-623.991016 Hartree/Particle

-624.050548 Hartree/Particle

$0.889 \mathrm{kcal} / \mathrm{mol}$

$0.889 \mathrm{kcal} / \mathrm{mol}$

$41.756 \mathrm{Cal} / \mathrm{mol}$

$32.405 \quad \mathrm{Cal} / \mathrm{mol}$

-624.018145 Hartree/Particle

-622.202336 Hartree/Particle

-624.275746 Hartree/Particle

Structure: Complex 7a

Z-matrix (molden format)

zmat angstroms

c

c $1 \mathrm{cc} 2$

c $2 \operatorname{cc} 3 \quad 1 \operatorname{ccc} 3$

c $3 \operatorname{cc} 4 \quad 2 \operatorname{ccc} 4 \quad 1 \operatorname{dih} 4$

c $4 \operatorname{cc} 5 \quad 3 \operatorname{ccc} 5 \quad 2 \operatorname{dih} 5$

c 5 cc6 4 cec6 3 dih 6 


\begin{tabular}{|c|c|c|}
\hline c 6 cc 7 & $5 \operatorname{ccc} 7$ & $4 \operatorname{dih} 7$ \\
\hline о 7 oc8 & 6 occ8 & $5 \operatorname{dih} 8$ \\
\hline li 8 lio9 & 7 lioc9 & $6 \operatorname{dih} 9$ \\
\hline c $7 \mathrm{cc} 10$ & $6 \operatorname{ccc} 10$ & $5 \operatorname{dih} 10$ \\
\hline c $10 \mathrm{cc} 11$ & $7 \operatorname{ccc} 11$ & $6 \operatorname{dih} 11$ \\
\hline o 9 oli12 & 8 olio12 & $7 \operatorname{dih} 12$ \\
\hline c 12 co13 & 9 coli13 & $8 \operatorname{dih} 13$ \\
\hline c 13 cc 14 & $12 \operatorname{cco} 14$ & $9 \operatorname{dih} 14$ \\
\hline c 14 cc 15 & $13 \operatorname{ccc} 15$ & $12 \operatorname{dih} 15$ \\
\hline h 10 hc16 & 7 hcc 16 & $6 \operatorname{dih} 16$ \\
\hline h 13 hc17 & 12 hco 17 & $14 \operatorname{dih} 17$ \\
\hline h 13 hc18 & 12 hco 18 & $14 \operatorname{dih} 18$ \\
\hline h 14 hc19 & 12 hco 19 & $13 \operatorname{dih} 19$ \\
\hline h 15 hc 20 & 14 hcc 20 & $12 \operatorname{dih} 20$ \\
\hline h 15 hc 21 & 14 hcc 21 & $12 \operatorname{dih} 21$ \\
\hline h 15 hc 22 & 14 hcc22 & $12 \operatorname{dih} 22$ \\
\hline h 11 hc 23 & 10 hcc 23 & $7 \operatorname{dih} 23$ \\
\hline h 11 hc 24 & 10 hcc 24 & $7 \operatorname{dih} 24$ \\
\hline h 11 hc 25 & 10 hcc 25 & $7 \operatorname{dih} 25$ \\
\hline h 1 hc26 & 2 hcc 26 & $3 \operatorname{dih} 26$ \\
\hline h 2 hc27 & 3 hcc27 & $4 \operatorname{dih} 27$ \\
\hline h 3 hc 28 & 2 hcc 28 & $1 \operatorname{dih} 28$ \\
\hline h 4 hc29 & 3 hcc 29 & $2 \operatorname{dih} 29$ \\
\hline \multirow{2}{*}{$\begin{array}{l}\text { h } 5 \text { hc30 } \\
\text { variables }\end{array}$} & 4 hcc30 & $3 \operatorname{dih} 30$ \\
\hline & & \\
\hline \multicolumn{2}{|c|}{$\begin{array}{cl}\text { cc2 } & 1.397007\end{array}$} & \\
\hline \multicolumn{2}{|c|}{$\begin{array}{ll}\text { cc3 } & 1.398397\end{array}$} & \\
\hline \multicolumn{3}{|c|}{$\begin{array}{ll}\operatorname{ccc} 3 & 120.290\end{array}$} \\
\hline c4 & 98927 & \\
\hline & 9.074 & \\
\hline dih4 & .145 & \\
\hline $\operatorname{cc} 5$ & 96592 & \\
\hline $\operatorname{ccc} 5$ & 0.460 & \\
\hline $\operatorname{dih} 5$ & .094 & \\
\hline 1. & 07278 & \\
\hline 12 & 1.226 & \\
\hline
\end{tabular}




$\begin{array}{ll}\text { dih6 } & -0.319 \\ \text { cc7 } & 1.501766 \\ \text { ccc7 } & 122.722 \\ \text { dih7 } & 179.622 \\ \text { oc8 } & 1.324446 \\ \text { occ8 } & 115.300 \\ \text { dih8 } & -148.534 \\ \text { lio9 } & 1.652617 \\ \text { lioc9 } & 168.644 \\ \text { dih9 } & 53.520 \\ \text { cc10 } & 1.361581 \\ \text { ccc10 } & 120.409 \\ \text { dih10 } & 32.413 \\ \text { cc11 } & 1.501708 \\ \text { ccc11 } & 124.305 \\ \text { dih11 } & 179.698 \\ \text { oli12 } & 1.900235 \\ \text { olio12 } & 133.458 \\ \text { dih12 } & -3.659 \\ \text { co13 } & 1.461118 \\ \text { coli13 } & 115.043 \\ \text { dih13 } & -30.510 \\ \text { cc14 } & 1.467442 \\ \text { cco14 } & 60.060 \\ \text { dih14 } & 109.320 \\ \text { cc15 } & 1.503916 \\ \text { ccc15 } & 122.891 \\ \text { dih15 } & 102.712 \\ \text { hc16 } & 1.087028 \\ \text { hcc16 } & 118.488 \\ \text { dih16 } & 1.962 \\ \text { hc17 } & 1.088525 \\ \text { hco17 } & 114.322 \\ \text { dih17 } & -111.589 \\ \text { hc18 } & 1.088145 \\ \text { hco18 } & 113.060\end{array}$




\begin{tabular}{|c|c|}
\hline $\operatorname{dih} 18$ & 111.757 \\
\hline hc19 & 1.090250 \\
\hline hco19 & 112.006 \\
\hline $\operatorname{dih} 19$ & 108.619 \\
\hline hc20 & 1.094645 \\
\hline hec20 & 111.050 \\
\hline $\operatorname{dih} 20$ & 43.875 \\
\hline hc21 & 1.095848 \\
\hline hec21 & 110.746 \\
\hline $\operatorname{dih} 21$ & -76.893 \\
\hline hc22 & 1.096781 \\
\hline hcc22 & 109.722 \\
\hline $\operatorname{dih} 22$ & 164.062 \\
\hline hc23 & 1.101339 \\
\hline hcc23 & 112.006 \\
\hline $\operatorname{dih} 23$ & -117.775 \\
\hline hc24 & 1.095070 \\
\hline hcc24 & 110.712 \\
\hline $\operatorname{dih} 24$ & 2.396 \\
\hline hc 25 & 1.101513 \\
\hline hcc 25 & 111.965 \\
\hline $\operatorname{dih} 25$ & 122.869 \\
\hline hc26 & 1.086563 \\
\hline hec26 & 120.133 \\
\hline $\operatorname{dih} 26$ & 179.269 \\
\hline hc27 & 1.088458 \\
\hline hec 27 & 119.966 \\
\hline $\operatorname{dih} 27$ & 179.429 \\
\hline hc28 & 1.087404 \\
\hline hcc28 & 120.481 \\
\hline $\operatorname{dih} 28$ & 179.652 \\
\hline hc29 & 1.087990 \\
\hline hec29 & 119.973 \\
\hline $\operatorname{dih} 29$ & 179.195 \\
\hline hc30 & 1.086503 \\
\hline hcc30 & 119.473 \\
\hline
\end{tabular}


constants

end

Number of imaginary frequency: 0

Thermochemical Data

Vacuum B3LYP Data

Electronic Energy=

Zero-point correction=

Thermal correction to Energy=

Thermal correction to Enthalpy=

Thermal correction to Gibbs Free Energy=

Sum of electronic and zero-point Energies=

Sum of electronic and thermal Energies=

Sum of electronic and thermal Enthalpies=

Sum of electronic and thermal Free Energies=

Thermal translational Energy=

Thermal rotational Energy=

Translational Entropy=

Rotational Entropy=

Corrected Free Energy= Vacuum MP2 data

Electronic Energy= IEF-PCM Data $(\varepsilon=2.104)$ $<\operatorname{Psi}(\mathrm{f})|\mathrm{H}+\mathrm{V}(\mathrm{f}) / 2| \operatorname{Psi}(\mathrm{f})>=$

Structure: Complex $7 b$

Z-matrix (molden format)
-624.325897 Hartree/Particle

0.243162 Hartree/Particle

0.259864 Hartree/Particle

0.260808 Hartree/Particle

0.193597 Hartree/Particle

-624.082735 Hartree/Particle

-624.066034 Hartree/Particle

-624.065090 Hartree/Particle

-624.132301 Hartree/Particle

$0.889 \quad \mathrm{kcal} / \mathrm{mol}$

$0.889 \quad \mathrm{kcal} / \mathrm{mol}$

$41.756 \quad \mathrm{Cal} / \mathrm{mol}$

$32.832 \quad \mathrm{Cal} / \mathrm{mol}$

-624.099695 Hartree/Particle

-622.281089 Hartree/Particle

-624.338764 Hartree/Particle 


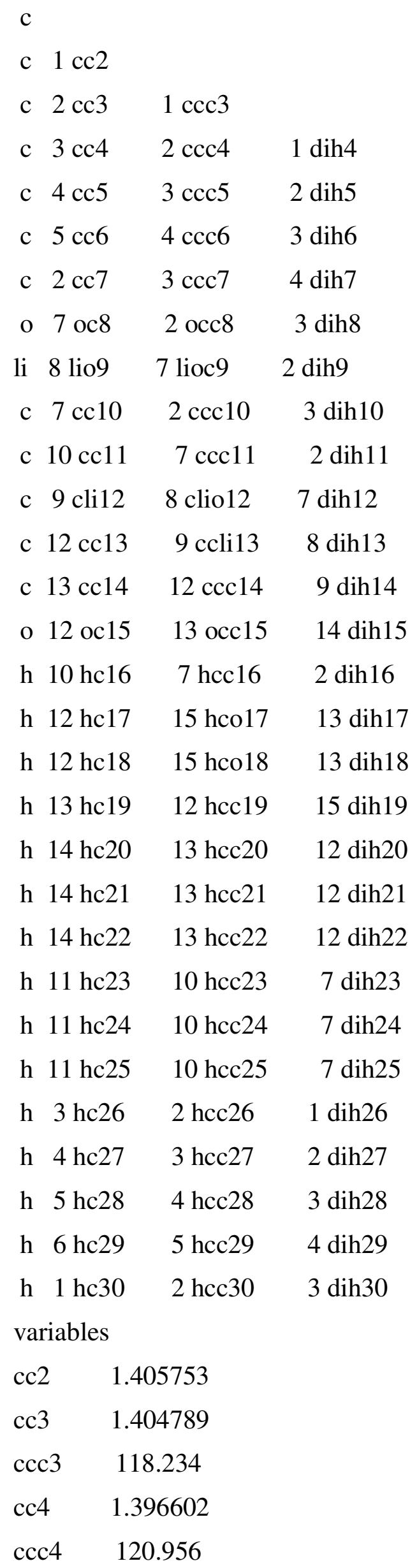




$\begin{array}{ll}\text { dih4 } & 1.259 \\ \text { cc5 } & 1.397862 \\ \text { ccc5 } & 120.236 \\ \text { dih5 } & -0.356 \\ \text { cc6 } & 1.398965 \\ \text { ccc6 } & 119.387 \\ \text { dih6 } & -0.505 \\ \text { cc7 } & 1.504604 \\ \text { ccc7 } & 118.688 \\ \text { dih7 } & 179.616 \\ \text { oc8 } & 1.316693 \\ \text { occ8 } & 116.863 \\ \text { dih8 } & -12.538 \\ \text { lio9 } & 1.753437 \\ \text { lioc9 } & 83.745 \\ \text { dih9 } & 129.487 \\ \text { cc10 } & 1.385169 \\ \text { ccc10 } & 121.359 \\ \text { dih10 } & 161.943 \\ \text { cc11 } & 1.517475 \\ \text { ccc11 } & 123.414 \\ \text { dih11 } & 165.650 \\ \text { cli12 } & 2.374545 \\ \text { clio12 } & 152.800 \\ \text { dih12 } & -120.644 \\ \text { cc13 } & 1.499889 \\ \text { ccli13 } & 74.247 \\ \text { dih13 } & -43.180 \\ \text { cc14 } & 1.509913 \\ \text { ccc14 } & 122.771 \\ \text { dih14 } & -82.919 \\ \text { oc15 } & 1.425332 \\ \text { occ15 } & 58.245 \\ \text { dih15 } & 103.452 \\ \text { hcc16 } & 1.085553 \\ & 119.238\end{array}$




\begin{tabular}{|c|c|}
\hline $\operatorname{dih} 16$ & -2.125 \\
\hline hc17 & 1.092530 \\
\hline hco17 & 114.692 \\
\hline $\operatorname{dih} 17$ & -112.289 \\
\hline hc18 & 1.092077 \\
\hline hco18 & 114.568 \\
\hline $\operatorname{dih} 18$ & 110.640 \\
\hline hc19 & 1.093343 \\
\hline hcc19 & 116.905 \\
\hline $\operatorname{dih} 19$ & -101.694 \\
\hline hc20 & 1.094968 \\
\hline hec20 & 111.133 \\
\hline $\operatorname{dih} 20$ & -21.882 \\
\hline hc21 & 1.095979 \\
\hline hcc21 & 109.560 \\
\hline $\operatorname{dih} 21$ & -141.497 \\
\hline hc22 & 1.097565 \\
\hline hec22 & 111.112 \\
\hline $\operatorname{dih} 22$ & 99.907 \\
\hline hc23 & 1.099643 \\
\hline hec23 & 110.645 \\
\hline $\operatorname{dih} 23$ & -122.681 \\
\hline hc24 & 1.099363 \\
\hline hec24 & 111.240 \\
\hline $\operatorname{dih} 24$ & -4.957 \\
\hline hc25 & 1.105392 \\
\hline hcc 25 & 114.939 \\
\hline $\operatorname{dih} 25$ & 118.763 \\
\hline hosk & 1.085248 \\
\hline hec26 & 118.225 \\
\hline $\operatorname{dih} 26$ & -177.862 \\
\hline hc27 & 1.087780 \\
\hline hec 27 & 119.741 \\
\hline $\operatorname{dih} 27$ & 179.949 \\
\hline hc28 & 1.087405 \\
\hline hcc28 & 120.363 \\
\hline
\end{tabular}




$\begin{array}{lc}\text { dih28 } & -179.762 \\ \text { hc29 } & 1.087627 \\ \text { hcc29 } & 120.075 \\ \text { dih29 } & -178.807 \\ \text { hc30 } & 1.086106 \\ \text { hcc30 } & 119.973 \\ \text { dih30 } & 177.215 \\ \text { constants } \\ \text { end }\end{array}$

Number of imaginary frequency: 0

Thermochemical Data

\section{Vacuum B3LYP Data}

Electronic Energy=

Zero-point correction=

Thermal correction to Energy=

Thermal correction to Enthalpy=

Thermal correction to Gibbs Free Energy=

Sum of electronic and zero-point Energies=

Sum of electronic and thermal Energies=

Sum of electronic and thermal Enthalpies=

Sum of electronic and thermal Free Energies=

Thermal translational Energy=

Thermal rotational Energy=

Translational Entropy=

Rotational Entropy=

Corrected Free Energy=

Vacuum MP2 data

Electronic Energy=

IEF-PCM Data $(\varepsilon=2.104)$

$<\operatorname{Psi}(\mathrm{f})|\mathrm{H}+\mathrm{V}(\mathrm{f}) / 2| \operatorname{Psi}(\mathrm{f})>=$

Structure: 1

$\begin{aligned}-624.299700 & \text { Hartree/Particle } \\ 0.242728 & \text { Hartree/Particle } \\ 0.259371 & \text { Hartree/Particle } \\ 0.260315 & \text { Hartree/Particle } \\ 0.194937 & \text { Hartree/Particle } \\ -624.056937 & \text { Hartree/Particle } \\ -624.040294 & \text { Hartree/Particle } \\ -624.039350 & \text { Hartree/Particle } \\ -624.104728 & \text { Hartree/Particle } \\ 0.889 & \text { Kcal/mol } \\ 0.889 & \text { Kcal/mol } \\ 41.756 & \text { Cal/mol } \\ 32.718 & \text { Cal } / \text { mol } \\ -624.072176 & \text { Hartree/Particle } \\ & \\ -622.255504 & \text { Hartree/Particle } \\ -624.308870 & \text { Hartree/Particle }\end{aligned}$




\section{zmat angstroms}

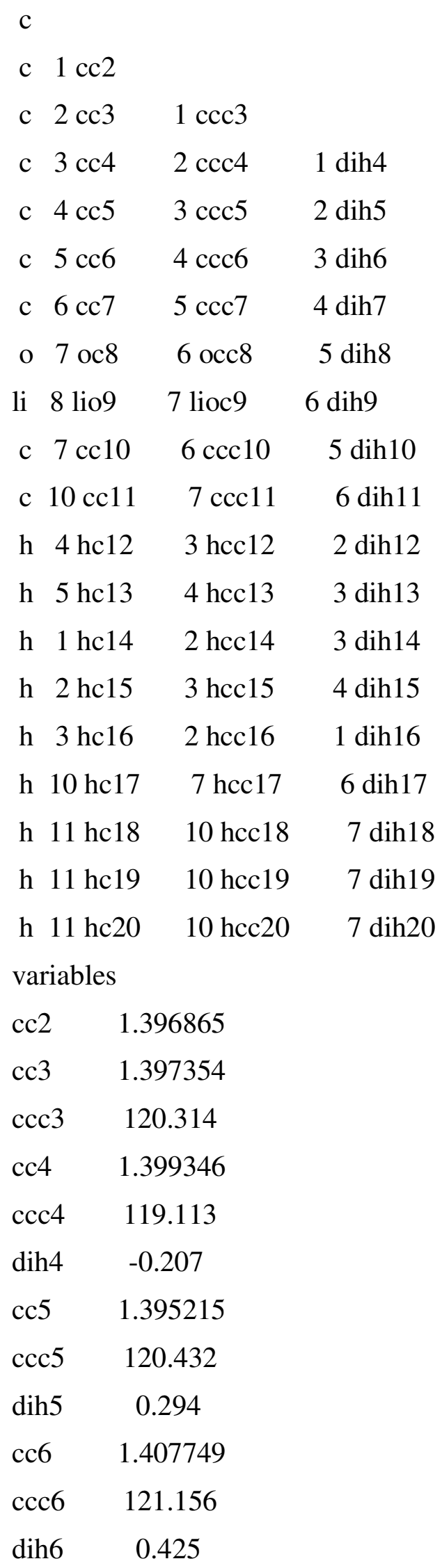




$\begin{array}{ll}\text { cc7 } & 1.496835 \\ \text { ccc7 } & 122.623 \\ \text { dih7 } & 179.300 \\ \text { oc8 } & 1.328032 \\ \text { occ8 } & 116.057 \\ \text { dih8 } & 157.377 \\ \text { lio9 } & 1.625374 \\ \text { lioc9 } & 167.487 \\ \text { dih9 } & -163.000 \\ \text { cc10 } & 1.359981 \\ \text { ccc10 } & 122.113 \\ \text { dih10 } & -23.886 \\ \text { cc11 } & 1.504231 \\ \text { ccc11 } & 122.533 \\ \text { dih11 } & -179.937 \\ \text { hc12 } & 1.087852 \\ \text { hcc12 } & 120.005 \\ \text { dih12 } & -178.937 \\ \text { hc13 } & 1.086064 \\ \text { hcc13 } & 119.232 \\ \text { dih13 } & -178.242 \\ \text { hc14 } & 1.085443 \\ \text { hcc14 } & 120.204 \\ \text { dih14 } & 179.964 \\ \text { hc15 } & 1.088146 \\ \text { hcc15 } & 119.996 \\ \text { dih15 } & 179.800 \\ \text { hc16 } & 1.087374 \\ \text { hcc16 } & 120.485 \\ \text { dih16 } & -179.674 \\ \text { hc17 } & 1.085216 \\ \text { hcc17 } & 119.486 \\ \text { dih17 } & -1.986 \\ \text { hc18 } & 1.096001 \\ \text { hcc18 } & 111.315 \\ \text { dih18 } & -176.884 \\ & \end{array}$


hc19 1.102137

hcc19 112.018

$\operatorname{dih} 19 \quad-56.700$

hc20 1.102663

hcc20 112.216

$\operatorname{dih} 20 \quad 62.968$

constants

end

Number of imaginary frequency: 0

Thermochemical Data

Vacuum B3LYP Data

Electronic Energy=

Zero-point correction=

Thermal correction to Energy=

Thermal correction to Enthalpy=

Thermal correction to Gibbs Free Energy=

Sum of electronic and zero-point Energies=

Sum of electronic and thermal Energies=

Sum of electronic and thermal Enthalpies=

Sum of electronic and thermal Free Energies=

Thermal translational Energy=

Thermal rotational Energy=

Translational Entropy=

Rotational Entropy=

Corrected Free Energy=

Vacuum MP2 data

Electronic Energy=

IEF-PCM Data $(\varepsilon=2.104)$

$<\operatorname{Psi}(\mathrm{f})|\mathrm{H}+\mathrm{V}(\mathrm{f}) / 2| \operatorname{Psi}(\mathrm{f})>=$
-431.172940 Hartree/Particle

0.155495 Hartree/Particle

0.166262 Hartree/Particle

0.167207 Hartree/Particle

0.117666 Hartree/Particle

-431.017445 Hartree/Particle

-431.006677 Hartree/Particle

-431.005733 Hartree/Particle

Hartree/Particle

$0.889 \quad \mathrm{kcal} / \mathrm{mol}$

$0.889 \quad \mathrm{kcal} / \mathrm{mol}$

$40.723 \quad \mathrm{Cal} / \mathrm{mol}$

$30.052 \quad \mathrm{Cal} / \mathrm{mol}$

-431.024480 Hartree/Particle

-429.750799 Hartree/Particle

-431.201137 Hartree/Particle

Structure: 2 
Z-matrix (molden format)

zmat angstroms

C

c $1 \mathrm{cc} 2$

c $2 \operatorname{cc} 3 \quad 1 \operatorname{ccc} 3$

o 3 oc4 2 occ $4 \quad 1$ dih4

h 3 hc5 2 hcc5 1 dih5

h 2 hc6 4 hco6 3 dih6

h 1 hc7 2 hcc7 $4 \operatorname{dih} 7$

h 1 hc8 2 hcc8 8 dih 8

h 1 hc9 2 hcc9 4 dih9

h 3 hc10 2 hcc10 1 dih10

variables

\begin{tabular}{lc} 
cc2 & 1.507988 \\
cc3 & 1.471182 \\
ccc3 & 122.627 \\
oc4 & 1.437818 \\
occ4 & 59.287 \\
dih4 & -103.911 \\
hc5 & 1.090497 \\
hcc5 & 119.398 \\
dih5 & -0.849 \\
hc6 & 1.092520 \\
hco6 & 112.893 \\
dih6 & -108.864 \\
hc7 & 1.096617 \\
hcc7 & 110.566 \\
dih7 & 75.640 \\
hc8 & 1.097472 \\
hcc8 & 110.463 \\
dih8 & -164.841 \\
hc9 & 1.095239 \\
hcc9 & 110.815 \\
dih9 & -44.372 \\
\hline
\end{tabular}


hc10 1.090114

hcc10 120.230

$\operatorname{dih} 10 \quad 153.211$

constants

end

Number of imaginary frequency: 0

Thermochemical Data

\section{Vacuum B3LYP Data}

Electronic Energy=

Zero-point correction $=$

Thermal correction to Energy=

Thermal correction to Enthalpy=

Thermal correction to Gibbs Free Energy=

Sum of electronic and zero-point Energies=

Sum of electronic and thermal Energies=

Sum of electronic and thermal Enthalpies=

Sum of electronic and thermal Free Energies=

Thermal translational Energy=

Thermal rotational Energy=

Translational Entropy=

Rotational Entropy=

Corrected Free Energy=

Vacuum MP2 data

Electronic Energy=

IEF-PCM Data $(\varepsilon=2.104)$

$<$ Psi(f) $|\mathrm{H}+\mathrm{V}(\mathrm{f}) / 2| \mathrm{Psi}(\mathrm{f})>=$

Structure: TS'

Z-matrix (molden format)
-193.118131 Hartree/Particle

0.085717 Hartree/Particle

0.090124 Hartree/Particle

0.091069 Hartree/Particle

0.059341 Hartree/Particle

-193.032414 Hartree/Particle

-193.028007 Hartree/Particle

-193.027062 Hartree/Particle

Hartree/Particle

$0.889 \mathrm{kcal} / \mathrm{mol}$

$0.889 \mathrm{kcal} / \mathrm{mol}$

$38.097 \quad \mathrm{Cal} / \mathrm{mol}$

$23.640 \quad \mathrm{Cal} / \mathrm{mol}$

-193.032290 Hartree/Particle

-192.490167 Hartree/Particle

-193.122147 Hartree/Particle 


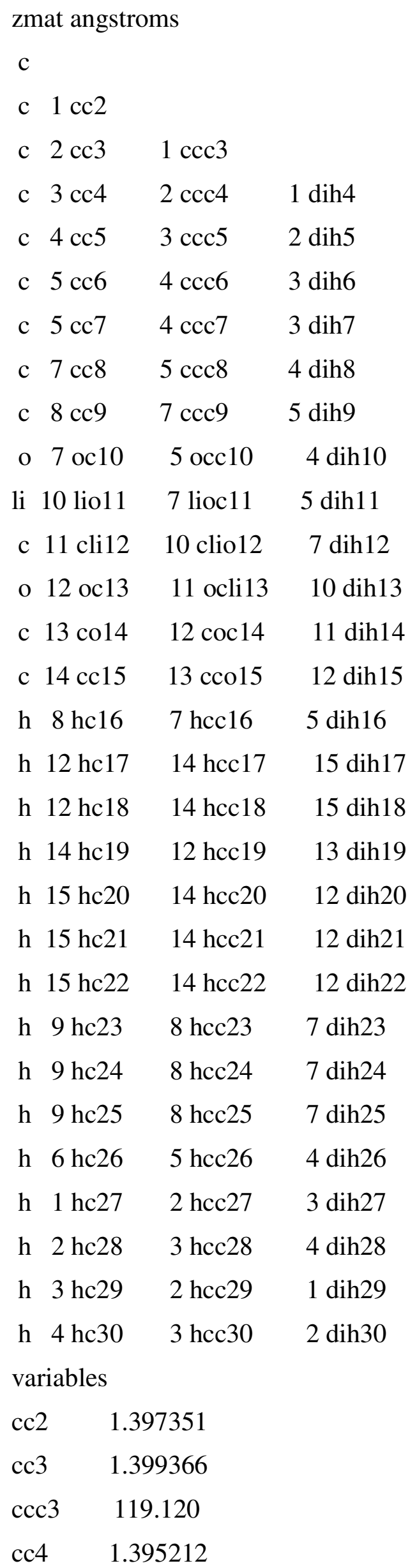




$\begin{array}{ll}\text { ccc4 } & 120.422 \\ \text { dih4 } & -0.288 \\ \text { cc5 } & 1.407768 \\ \text { ccc5 } & 121.156 \\ \text { dih5 } & -0.408 \\ \text { cc6 } & 1.405758 \\ \text { ccc6 } & 117.693 \\ \text { dih6 } & 1.158 \\ \text { cc7 } & 1.497158 \\ \text { ccc7 } & 122.660 \\ \text { dih7 } & -179.365 \\ \text { cc8 } & 1.360337 \\ \text { ccc8 } & 122.105 \\ \text { dih8 } & 23.474 \\ \text { cc9 } & 1.504209 \\ \text { ccc9 } & 122.489 \\ \text { dih9 } & 179.976 \\ \text { oc10 } & 1.327044 \\ \text { occ10 } & 116.046 \\ \text { dih10 } & -157.703 \\ \text { lio11 } & 1.622940 \\ \text { lioc11 } & 167.712 \\ \text { dih11 } & 163.248 \\ \text { cli12 } & 2.481498 \\ \text { clio12 } & 163.765 \\ \text { dih12 } & -13.743 \\ \text { oc13 } & 1.423396 \\ \text { ocli13 } & 161.549 \\ \text { dih13 } & -164.349 \\ \text { co14 } & 1.443366 \\ \text { coc14 } & 61.845 \\ \text { dih14 } & -175.167 \\ \text { cc15 } & 1.505935 \\ \text { cco15 } & 117.011 \\ \text { dih15 } & -113.164 \\ \text { hc16 } & 1.085196\end{array}$




$\begin{array}{lc}\text { hcc16 } & 119.524 \\ \text { dih16 } & 2.114 \\ \text { hc17 } & 1.097946 \\ \text { hcc17 } & 119.377 \\ \text { dih17 } & -154.222 \\ \text { hc18 } & 1.096787 \\ \text { hcc18 } & 118.852 \\ \text { dih18 } & 1.440 \\ \text { hc19 } & 1.091157 \\ \text { hcc19 } & 116.603 \\ \text { dih19 } & -101.540 \\ \text { hc20 } & 1.094863 \\ \text { hcc20 } & 111.079 \\ \text { dih20 } & -25.222 \\ \text { hc21 } & 1.095767 \\ \text { hcc21 } & 110.231 \\ \text { dih21 } & -145.240 \\ \text { hc22 } & 1.096608 \\ \text { hcc22 } & 110.327 \\ \text { dih22 } & 95.486 \\ \text { hc23 } & 1.102779 \\ \text { hcc23 } & 112.247 \\ \text { dih23 } & -64.213 \\ \text { hc24 } & 1.102112 \\ \text { hcc24 } & 111.945 \\ \text { dih24 } & 55.381 \\ \text { hc25 } & 1.096113 \\ \text { hcc25 } & 111.322 \\ \text { dih25 } & 175.603 \\ \text { hc26 } & 1.085415 \\ \text { hcc26 } & 118.505 \\ \text { dih26 } & 179.259 \\ \text { hc27 } & 1.088127 \\ \text { hcc27 } & 119.996 \\ \text { dih27 } & -179.811 \\ \text { hc28 } & 1.087380 \\ & \end{array}$




$\begin{array}{lc}\text { hcc28 } & 120.398 \\ \operatorname{dih} 28 & -179.772 \\ \text { hc29 } & 1.087861 \\ \text { hcc29 } & 120.011 \\ \text { dih29 } & 178.946 \\ \text { hc30 } & 1.086100 \\ \text { hcc30 } & 119.229 \\ \text { dih30 } & 178.260 \\ \text { constants } \\ \text { end }\end{array}$

Number of imaginary frequency: 1 (eigenvalue $=-67.1556)$

Thermochemical Data

Vacuum B3LYP Data

Electronic Energy=

Zero-point correction=

Thermal correction to Energy=

Thermal correction to Enthalpy=

Thermal correction to Gibbs Free Energy=

Sum of electronic and zero-point Energies=

Sum of electronic and thermal Energies=

Sum of electronic and thermal Enthalpies=

Sum of electronic and thermal Free Energies= -624.103281 Hartree/Particle

Thermal translational Energy=

Thermal rotational Energy=

Translational Entropy=

Rotational Entropy=

Corrected Free Energy=

Vacuum MP2 data

Electronic Energy=

IEF-PCM Data $(\varepsilon=2.104)$

$<\operatorname{Psi}(\mathrm{f})|\mathrm{H}+\mathrm{V}(\mathrm{f}) / 2| \mathrm{Psi}(\mathrm{f})>=$
-624.292496 Hartree/Particle

0.241618 Hartree/Particle

0.258206 Hartree/Particle

0.259150 Hartree/Particle

0.189214 Hartree/Particle

-624.050878 Hartree/Particle

-624.034289 Hartree/Particle

-624.033345 Hartree/Particle

$0.889 \mathrm{kcal} / \mathrm{mol}$

$0.889 \mathrm{kcal} / \mathrm{mol}$

$41.756 \quad \mathrm{Cal} / \mathrm{mol}$

$33.416 \quad \mathrm{Cal} / \mathrm{mol}$

-624.070398 Hartree/Particle

-622.243224 Hartree/Particle

-624.308632 Hartree/Particle 
Structure: intermediate I

Z-matrix (molden format)

zmat angstroms

c

c $1 \mathrm{cc} 2$

c $2 \operatorname{cc} 3 \quad 1 \operatorname{ccc} 3$

c $3 \operatorname{cc} 4 \quad 2 \operatorname{ccc} 4 \quad 1 \operatorname{dih} 4$

c $4 \operatorname{cc} 5 \quad 3 \operatorname{ccc} 5 \quad 2$ dih5

c $5 \operatorname{cc} 6 \quad 4 \operatorname{ccc} 6 \quad 3 \operatorname{dih} 6$

$\begin{array}{lll}\text { c } 5 \operatorname{cc} 7 & 4 \operatorname{ccc} 7 & 3 \operatorname{dih} 7\end{array}$

c $7 \operatorname{cc} 8 \quad 5 \operatorname{ccc} 8 \quad 4 \operatorname{dih} 8$

c $8 \operatorname{cc} 9 \quad 7 \operatorname{ccc} 9 \quad 5$ dih 9

o 7 oc10 5 occ10 4 dih10

li 10 lio11 7 lioc11 5 dih11

c 11 cli12 10 clio12 7 dih12

o 12 oc13 11 ocli13 10 dih13

c 13 col4 12 coc $14 \quad 11 \operatorname{dih} 14$

c $14 \operatorname{cc} 15 \quad 13 \operatorname{cco} 15 \quad 12 \operatorname{dih} 15$

h 8 hc16 7 hcc16 5 dih16

h 12 hc17 14 hcc17 15 dih17

h 12 hc18 14 hcc18 $15 \operatorname{dih} 18$

h 14 hc19 12 hcc19 13 dih19

h 15 hc20 14 hcc20 $12 \operatorname{dih} 20$

h 15 hc21 14 hcc21 12 dih 21

h 15 hc22 14 hcc $22 \quad 12 \operatorname{dih} 22$

h 9 hc23 8 hcc23 $7 \operatorname{dih} 23$

h 9 hc24 8 hcc24 7 dih 24

h 9 hc25 8 hcc25 $7 \operatorname{dih} 25$

h 6 hc26 5 hcc26 4 dih 26

h 1 hc27 2 hcc27 $3 \operatorname{dih} 27$

h 2 hc28 3 hcc $28 \quad 4 \operatorname{dih} 28$

h 3 hc29 2 hcc29 1 dih29

h 4 hc30 3 hcc30 2 dih 30 


$\begin{array}{ll}\text { variables } \\ \text { cc2 } & 1.397481 \\ \text { cc3 } & 1.399369 \\ \text { ccc3 } & 119.107 \\ \text { cc4 } & 1.395373 \\ \text { ccc4 } & 120.437 \\ \text { dih4 } & -0.270 \\ \text { cc5 } & 1.407806 \\ \text { ccc5 } & 121.163 \\ \text { dih5 } & -0.406 \\ \text { cc6 } & 1.406016 \\ \text { ccc6 } & 117.664 \\ \text { dih6 } & 1.163 \\ \text { cc7 } & 1.497541 \\ \text { ccc7 } & 122.660 \\ \text { dih7 } & -179.423 \\ \text { cc8 } & 1.360265 \\ \text { ccc8 } & 121.971 \\ \text { dih8 } & 24.701 \\ \text { cc9 } & 1.504346 \\ \text { ccc9 } & 122.518 \\ \text { dih9 } & 179.773 \\ \text { oc10 } & 1.326871 \\ \text { occ10 } & 116.014 \\ \text { dih10 } & -156.509 \\ \text { lio11 } & 1.627601 \\ \text { lioc11 } & 169.236 \\ \text { dih11 } & 159.255 \\ \text { cli12 } & 2.363739 \\ \text { clio12 } & 169.886 \\ \text { dih12 } & -157.203 \\ \text { oc13 } & 1.423430 \\ \text { ocli13 } & 129.302 \\ & 100.269 \\ \text { dih } & 63.739\end{array}$




\begin{tabular}{|c|c|}
\hline $\operatorname{dih} 14$ & -9.872 \\
\hline cc15 & 1.510641 \\
\hline $\operatorname{cco} 15$ & 117.244 \\
\hline $\operatorname{dih} 15$ & -113.169 \\
\hline hc16 & 1.085287 \\
\hline hcc16 & 119.474 \\
\hline $\operatorname{dih} 16$ & 1.926 \\
\hline hc17 & 1.093648 \\
\hline hcc17 & 121.040 \\
\hline $\operatorname{dih} 17$ & -154.783 \\
\hline hc18 & 1.091865 \\
\hline hec18 & 119.301 \\
\hline $\operatorname{dih} 18$ & 1.427 \\
\hline hc19 & 1.095157 \\
\hline hcc19 & 117.028 \\
\hline $\operatorname{dih} 19$ & -100.962 \\
\hline hc20 & 1.094480 \\
\hline hec20 & 110.822 \\
\hline $\operatorname{dih} 20$ & -24.530 \\
\hline hc21 & 1.095713 \\
\hline hec21 & 109.525 \\
\hline $\operatorname{dih} 21$ & -144.074 \\
\hline hc22 & 1.097894 \\
\hline hec22 & 111.327 \\
\hline $\operatorname{dih} 22$ & 96.890 \\
\hline hc23 & 1.102341 \\
\hline hcc23 & 112.129 \\
\hline $\operatorname{dih} 23$ & -61.387 \\
\hline hc24 & 1.102054 \\
\hline hec24 & 112.095 \\
\hline $\operatorname{dih} 24$ & 58.153 \\
\hline hc25 & 1.096028 \\
\hline hec25 & 111.307 \\
\hline $\operatorname{dih} 25$ & 178.402 \\
\hline hc26 & 1.085569 \\
\hline hcc26 & 118.478 \\
\hline
\end{tabular}




$\begin{array}{lc}\text { dih26 } & 179.399 \\ \text { hc27 } & 1.088196 \\ \text { hcc27 } & 119.988 \\ \text { dih27 } & -179.920 \\ \text { hc28 } & 1.087368 \\ \text { hcc28 } & 120.408 \\ \text { dih28 } & -179.792 \\ \text { hc29 } & 1.087861 \\ \text { hcc29 } & 120.001 \\ \text { dih29 } & 178.958 \\ \text { hc30 } & 1.086159 \\ \text { hcc30 } & 119.262 \\ \text { dih30 } & 178.263 \\ \text { constants } & \\ \text { end } & \end{array}$

Number of imaginary frequency: 0

Thermochemical Data 
Vacuum B3LYP Data

$\begin{array}{lrl}\text { Electronic Energy= } & -624.299555 & \text { Hartree/Particle } \\ \text { Zero-point correction= } & 0.242065 & \text { Hartree/Particle } \\ \text { Thermal correction to Energy= } & 0.259288 & \text { Hartree/Particle } \\ \text { Thermal correction to Enthalpy= } & 0.260232 & \text { Hartree/Particle } \\ \text { Thermal correction to Gibbs Free Energy= } & 0.191175 & \text { Hartree/Particle } \\ \text { Sum of electronic and zero-point Energies= } & -624.057490 & \text { Hartree/Particle } \\ \text { Sum of electronic and thermal Energies= } & -624.040267 & \text { Hartree/Particle } \\ \text { Sum of electronic and thermal Enthalpies }= & -624.039323 & \text { Hartree/Particle } \\ \text { Sum of electronic and thermal Free Energies= }=-624.108380 & \text { Hartree/Particle } \\ \text { Thermal translational Energy= } & 0.889 & \text { kcal/mol } \\ \text { Thermal rotational Energy= } & 0.889 & \text { kcal/mol } \\ \text { Translational Entropy= } & 41.756 & \text { Cal/mol } \\ \text { Rotational Entropy= } & 33.091 & \text { Cal/mol } \\ \text { Corrected Free Energy= } & -624.075651 & \text { Hartree/Particle } \\ \quad \text { Vacuum MP2 data } & & \\ \text { Electronic Energy= } & -622.253223 & \text { Hartree/Particle } \\ \text { IEF-PCM Data }(\varepsilon=2.104) & & \\ <\text { Psi(f)|H+V(f)/2|Psi(f) }>= & \text { Hartree/Particle }\end{array}$

Structure: TS“

Z-matrix (molden format)

zmat angstroms

$\begin{array}{lll}\text { c } & & \\ \text { c } 1 \text { cc } 2 & & \\ \text { c } 2 \operatorname{cc} 3 & 1 \operatorname{ccc} 3 & \\ \text { c } 3 \operatorname{cc} 4 & 2 \operatorname{ccc} 4 & 1 \operatorname{dih} 4 \\ \text { c } 4 \operatorname{cc} 5 & 3 \operatorname{ccc} 5 & 2 \operatorname{dih} 5 \\ \text { c } 5 \text { cc6 } & 4 \operatorname{ccc} 6 & 3 \operatorname{dih} 6 \\ \text { c } 5 \text { cc7 } & 4 \operatorname{ccc} 7 & 3 \operatorname{dih} 7 \\ \text { c } 7 \operatorname{cc} 8 & 5 \operatorname{ccc} 8 & 4 \operatorname{dih} 8 \\ \text { c } 8 \operatorname{cc} 9 & 7 \operatorname{ccc} 9 & 5 \operatorname{dih} 9\end{array}$




\begin{tabular}{|c|c|c|}
\hline o 7 oc10 & 5 occ 10 & $4 \operatorname{dih} 10$ \\
\hline li 10 lio1 1 & 7 lioc11 & $5 \operatorname{dih} 11$ \\
\hline c 11 cli 12 & 10 clio12 & $7 \operatorname{dih} 12$ \\
\hline o 12 oc 13 & 11 ocli13 & $10 \operatorname{dih} 13$ \\
\hline c 13 co14 & $12 \operatorname{coc} 14$ & $11 \operatorname{dih} 14$ \\
\hline c $14 \operatorname{cc} 15$ & 13 cco 15 & $12 \operatorname{dih} 15$ \\
\hline h 8 hc16 & 7 hcc16 & $5 \operatorname{dih} 16$ \\
\hline h 12 hc17 & 14 hcc17 & $15 \operatorname{dih} 17$ \\
\hline h 12 hc18 & 14 hcc18 & $15 \operatorname{dih} 18$ \\
\hline h 14 hc19 & 13 hco19 & $12 \operatorname{dih} 19$ \\
\hline h 15 hc 20 & 14 hcc 20 & $13 \operatorname{dih} 20$ \\
\hline h 15 hc 21 & 14 hcc 21 & $13 \operatorname{dih} 21$ \\
\hline h 15 hc 22 & 14 hcc 22 & $13 \operatorname{dih} 22$ \\
\hline h 9 hc 23 & 8 hcc 23 & $7 \operatorname{dih} 23$ \\
\hline h 9 hc24 & 8 hcc24 & $7 \operatorname{dih} 24$ \\
\hline h 9 hc 25 & 8 hcc 25 & $7 \operatorname{dih} 25$ \\
\hline h 6 hc26 & 5 hcc 26 & $4 \operatorname{dih} 26$ \\
\hline h 1 hc 27 & 2 hcc 27 & $3 \operatorname{dih} 27$ \\
\hline h 2 hc 28 & 3 hcc 28 & $4 \operatorname{dih} 28$ \\
\hline h 3 hc29 & 2 hcc 29 & $1 \operatorname{dih} 29$ \\
\hline \multirow{2}{*}{$\begin{array}{l}\text { h } 4 \text { hc30 } \\
\text { variables }\end{array}$} & 3 hcc30 & $2 \operatorname{dih} 30$ \\
\hline & & \\
\hline \multicolumn{2}{|c|}{1.397414} & \\
\hline \multicolumn{2}{|c|}{1.399117} & \\
\hline $\operatorname{ccc} 3$ & .276 & \\
\hline$c 4$ & 5592 & \\
\hline $\operatorname{ccc} 4$ & .338 & \\
\hline $\operatorname{dih} 4$ & 428 & \\
\hline $\operatorname{cc} 5$ & 6349 & \\
\hline $\operatorname{ccc} 5$ & .945 & \\
\hline $\operatorname{dih} 5$ & 037 & \\
\hline $\operatorname{cc} 6$ & 4714 & \\
\hline $\operatorname{ccc} 6$ & .125 & \\
\hline $\operatorname{dih} 6$ & 858 & \\
\hline 1.5 & 1123 & \\
\hline $\operatorname{ccc} 7$ & .468 & \\
\hline
\end{tabular}




$\begin{array}{lc}\text { dih7 } & -179.404 \\ \text { cc8 } & 1.374122 \\ \text { ccc8 } & 121.527 \\ \text { dih8 } & 18.520 \\ \text { cc9 } & 1.512770 \\ \text { ccc9 } & 123.045 \\ \text { dih9 } & 173.902 \\ \text { oc10 } & 1.324731 \\ \text { occ10 } & 116.024 \\ \text { dih10 } & -161.777 \\ \text { lio11 } & 1.728284 \\ \text { lioc11 } & 99.881 \\ \text { dih11 } & 145.246 \\ \text { cli12 } & 2.372182 \\ \text { clio12 } & 144.771 \\ \text { dih12 } & -168.872 \\ \text { oc13 } & 1.423661 \\ \text { ocli13 } & 130.699 \\ \text { dih13 } & 34.640 \\ \text { co14 } & 1.427297 \\ \text { coc14 } & 63.587 \\ \text { dih14 } & -13.809 \\ \text { cc15 } & 1.508699 \\ \text { cco15 } & 117.495 \\ \text { dih15 } & -113.031 \\ \text { hc16 } & 1.084041 \\ \text { hcc16 } & 120.198 \\ \text { dih16 } & -0.467 \\ \text { hc17 } & 1.094519 \\ \text { hcc17 } & 121.300 \\ \text { dih17 } & -154.707 \\ \text { hc18 } & 1.091766 \\ \text { hcc18 } & 119.361 \\ \text { dih18 } & 1.611 \\ \text { hc19 } & 1.094061 \\ \text { hco19 } & 112.733 \\ & \end{array}$




\begin{tabular}{|c|c|}
\hline $\operatorname{dih} 19$ & 108.665 \\
\hline hc20 & 1.094959 \\
\hline hcc20 & 110.880 \\
\hline $\operatorname{dih} 20$ & 44.051 \\
\hline hc21 & 1.095976 \\
\hline hcc21 & 109.460 \\
\hline $\operatorname{dih} 21$ & -75.351 \\
\hline hc22 & 1.096415 \\
\hline hec22 & 110.417 \\
\hline $\operatorname{dih} 22$ & 165.652 \\
\hline hc23 & 1.095848 \\
\hline hec23 & 111.306 \\
\hline $\operatorname{dih} 23$ & -150.641 \\
\hline hc24 & 1.104068 \\
\hline hcc24 & 111.603 \\
\hline $\operatorname{dih} 24$ & -32.472 \\
\hline hc 25 & 1.114276 \\
\hline hec25 & 115.617 \\
\hline $\operatorname{dih} 25$ & 91.547 \\
\hline hc26 & 1.084971 \\
\hline hec26 & 118.162 \\
\hline $\operatorname{dih} 26$ & 178.978 \\
\hline hc27 & 1.087850 \\
\hline hec27 & 120.009 \\
\hline $\operatorname{dih} 27$ & -179.776 \\
\hline hc 28 & 1.087390 \\
\hline hec28 & 120.284 \\
\hline $\operatorname{dih} 28$ & 179.991 \\
\hline hc29 & 1.087723 \\
\hline hec29 & 120.055 \\
\hline $\operatorname{dih} 29$ & 179.139 \\
\hline hc30 & 1.086185 \\
\hline hec30 & 119.003 \\
\hline $\operatorname{dih} 30$ & 178.945 \\
\hline Jist & \\
\hline & \\
\hline
\end{tabular}


Number of imaginary frequency: 1 (eigenvalue $=-126.7186$ )

Thermochemical Data

Vacuum B3LYP Data

Electronic Energy=

Zero-point correction=

Thermal correction to Energy=

Thermal correction to Enthalpy=

Thermal correction to Gibbs Free Energy=

Sum of electronic and zero-point Energies=

Sum of electronic and thermal Energies=

Sum of electronic and thermal Enthalpies=

Sum of electronic and thermal Free Energies=

Thermal translational Energy=

Thermal rotational Energy=

Translational Entropy=

Rotational Entropy=

Corrected Free Energy=

Vacuum MP2 data

Electronic Energy=

IEF-PCM Data $(\varepsilon=2.104)$

$<\operatorname{Psi}(\mathrm{f})|\mathrm{H}+\mathrm{V}(\mathrm{f}) / 2| \operatorname{Psi}(\mathrm{f})>=$

Structure: $3 \mathrm{a}$

Z-matrix (molden format)

zmat angstroms

c

c $1 \mathrm{cc} 2$

c $2 \operatorname{cc} 3 \quad 1 \operatorname{ccc} 3$

c $3 \operatorname{cc} 4 \quad 2 \operatorname{ccc} 4 \quad 1 \operatorname{dih} 4$
-624.297152 Hartree/Particle

$0.241915 \quad$ Hartree/Particle

$0.257889 \quad$ Hartree/Particle

$0.258833 \quad$ Hartree/Particle

$0.194880 \quad$ Hartree/Particle

-624.055237 Hartree/Particle

-624.039263 Hartree/Particle

$-624.038319 \quad$ Hartree/Particle

-624.102271 Hartree/Particle

$0.889 \quad \mathrm{kcal} / \mathrm{mol}$

$0.889 \quad \mathrm{kcal} / \mathrm{mol}$

$41.756 \quad \mathrm{Cal} / \mathrm{mol}$

$32.797 \quad \mathrm{Cal} / \mathrm{mol}$

-624.069682 Hartree/Particle

$-622.252829 \quad$ Hartree/Particle

-624.307661 Hartree/Particle 


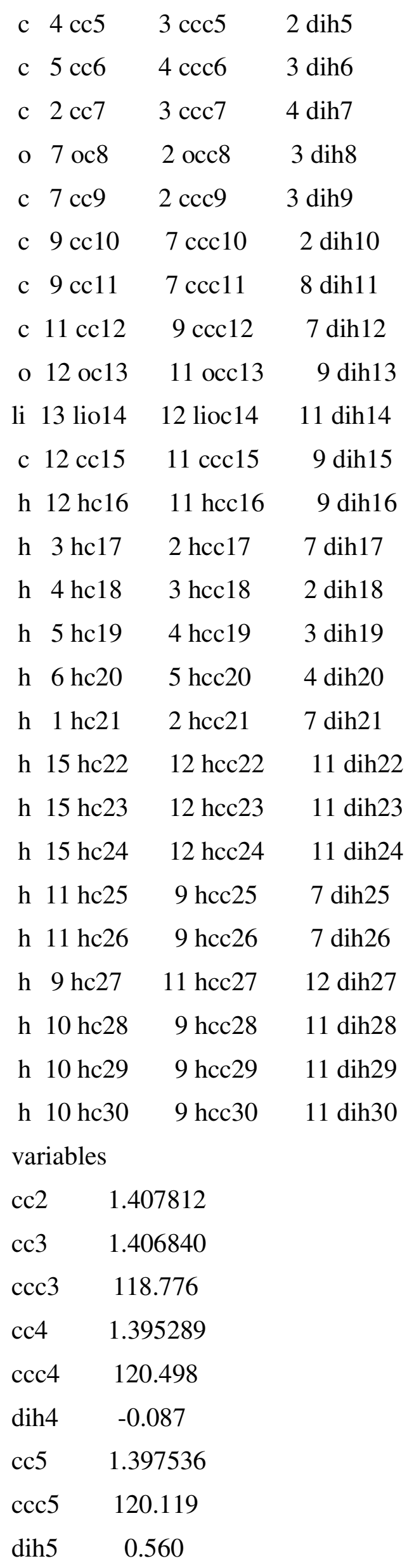




$\begin{array}{ll}\text { cc6 } & 1.399529 \\ \text { ccc6 } & 119.920 \\ \text { dih6 } & -0.405 \\ \text { cc7 } & 1.496012 \\ \text { ccc7 } & 122.886 \\ \text { dih7 } & 179.223 \\ \text { oc8 } & 1.243228 \\ \text { occ8 } & 118.117 \\ \text { dih8 } & -172.924 \\ \text { cc9 } & 1.522264 \\ \text { ccc9 } & 120.032 \\ \text { dih9 } & 7.953 \\ \text { cc10 } & 1.537370 \\ \text { ccc10 } & 112.171 \\ \text { dih10 } & 145.751 \\ \text { cc11 } & 1.573214 \\ \text { ccc11 } & 111.716 \\ \text { dih11 } & 95.219 \\ \text { cc12 } & 1.555233 \\ \text { ccc12 } & 115.984 \\ \text { dih12 } & -87.616 \\ \text { oc13 } & 1.373655 \\ \text { occ13 } & 112.675 \\ \text { dih13 } & 53.756 \\ \text { lio14 } & 1.670063 \\ \text { lioc14 } & 138.412 \\ \text { dih14 } & -33.726 \\ \text { cc15 } & 1.542226 \\ \text { ccc15 } & 108.671 \\ \text { dih15 } & 178.280 \\ \text { hc16 } & 1.113811 \\ \text { hcc16 } & 106.293 \\ \text { dih16 } & -67.899 \\ \text { hc17 } & 1.084401 \\ \text { hcc17 } & 120.585 \\ \text { dih17 } & 0.193 \\ & \end{array}$




\begin{tabular}{ll} 
hc18 & 1.086570 \\
hcc18 & 119.705 \\
dih18 & -179.439 \\
hc19 & 1.087020 \\
hcc19 & 120.011 \\
dih19 & 179.634 \\
hc20 & 1.086754 \\
hcc20 & 120.095 \\
dih20 & 179.951 \\
hc21 & 1.084949 \\
hcc21 & 118.629 \\
dih21 & 0.511 \\
hc22 & 1.095733 \\
hcc22 & 109.083 \\
dih22 & 177.879 \\
hc23 & 1.098304 \\
hcc23 & 110.126 \\
dih23 & -64.026 \\
hc24 & 1.099828 \\
hcc24 & 112.495 \\
dih24 & 57.453 \\
hc25 & 1.099327 \\
hcc25 & 109.299 \\
dih25 & 35.087 \\
hc26 & 1.100396 \\
hcc26 & 106.807 \\
dih26 & 150.211 \\
hc27 & 1.096179 \\
hcc27 & 106.248 \\
dih27 & 157.422 \\
hc28 & 1.096375 \\
hcc28 & 111.083 \\
dih28 & 177.848 \\
hc29 & 1.092759 \\
hcc29 & 111.993 \\
dih29 & -60.519 \\
& \\
\hline
\end{tabular}


hc30 $\quad 1.095515$

hcc30 109.344

$\operatorname{dih} 30 \quad 58.734$

constants

end

Number of imaginary frequency: 0

Thermochemical Data

\section{Vacuum B3LYP Data}

Electronic Energy=

Zero-point correction $=$

Thermal correction to Energy=

Thermal correction to Enthalpy=

Thermal correction to Gibbs Free Energy=

Sum of electronic and zero-point Energies=

Sum of electronic and thermal Energies=

Sum of electronic and thermal Enthalpies=

Sum of electronic and thermal Free Energies=

Thermal translational Energy=

Thermal rotational Energy=

Translational Entropy=

Rotational Entropy=

Corrected Free Energy=

Vacuum MP2 data

Electronic Energy=

IEF-PCM Data $(\varepsilon=2.104)$

$<\operatorname{Psi}(\mathrm{f})|\mathrm{H}+\mathrm{V}(\mathrm{f}) / 2| \mathrm{Psi}(\mathrm{f})>=$
-624.368881 Hartree/Particle

$0.247116 \quad$ Hartree/Particle

$0.261673 \quad$ Hartree/Particle

$0.262617 \quad$ Hartree/Particle

$0.205354 \quad$ Hartree/Particle

-624.121764 Hartree/Particle

-624.107207 Hartree/Particle

-624.106263 Hartree/Particle

-624.163526 Hartree/Particle

$0.889 \mathrm{kcal} / \mathrm{mol}$

$0.889 \mathrm{kcal} / \mathrm{mol}$

$41.756 \mathrm{Cal} / \mathrm{mol}$

$32.097 \quad \mathrm{Cal} / \mathrm{mol}$

-624.131269 Hartree/Particle

$-622.331027 \quad$ Hartree/Particle

-624.381360 Hartree/Particle

Structure: $3 b$

Z-matrix (molden format) 


\section{zmat angstroms}

c

c $1 \mathrm{cc} 2$

c $2 \operatorname{cc} 3 \quad 1 \operatorname{ccc} 3$

c $3 \operatorname{cc} 4 \quad 2 \operatorname{ccc} 4 \quad 1$ dih 4

c $4 \operatorname{cc} 5 \quad 3 \operatorname{ccc} 5 \quad 2 \operatorname{dih} 5$

c 5 cc6 4 ccc6 3 dih6

c $6 \operatorname{cc} 7 \quad 5 \operatorname{ccc} 7 \quad 4 \operatorname{dih} 7$

o 7 oc8 6 occ $8 \quad 5 \operatorname{dih} 8$

c 7 cc9 $6 \operatorname{ccc} 9 \quad 5 \operatorname{dih} 9$

c $9 \operatorname{cc} 10 \quad 7 \operatorname{ccc} 10 \quad 6 \operatorname{dih} 10$

c $9 \operatorname{cc} 11 \quad 7 \operatorname{ccc} 11 \quad 6 \operatorname{dih} 11$

c $11 \operatorname{cc} 12 \quad 9 \operatorname{ccc} 12 \quad 7 \operatorname{dih} 12$

o 12 oc13 11 occ13 $9 \operatorname{dih} 13$

li 13 lio14 12 lioc14 11 dih14

c $12 \operatorname{cc} 15 \quad 11 \operatorname{ccc} 15 \quad 9 \operatorname{dih} 15$

h 9 hc16 7 hcc16 6 dih16

h 11 hc17 9 hcc17 $\quad 7$ dih17

h 11 hc18 9 hcc18 $\quad 7$ dih18

h 12 hc19 11 hcc19 9 dih19

h 15 hc20 12 hcc $20 \quad 11 \operatorname{dih} 20$

h 15 hc21 12 hcc21 11 dih 21

h 15 hc22 12 hcc $22 \quad 11 \operatorname{dih} 22$

h 10 hc23 9 hcc23 7 dih23

h 10 hc24 9 hcc24 7 dih24

h 10 hc25 9 hcc $25 \quad 7$ dih 25

h 1 hc26 2 hcc26 $3 \operatorname{dih} 26$

h 2 hc27 3 hcc $27 \quad 4 \operatorname{dih} 27$

h 3 hc $28 \quad 2$ hcc $28 \quad 1 \operatorname{dih} 28$

h 4 hc29 3 hcc29 $2 \operatorname{dih} 29$

h 5 hc30 4 hcc30 $3 \operatorname{dih} 30$

variables

cc2 1.393117

cc3 1.399578

ccc3 120.057

cc4 1.396882 


\begin{tabular}{ll} 
ccc4 & 119.686 \\
dih4 & 0.046 \\
cc5 & 1.397183 \\
ccc5 & 120.200 \\
dih5 & 0.254 \\
cc6 & 1.404505 \\
ccc6 & 120.647 \\
dih6 & -0.314 \\
cc7 & 1.509005 \\
ccc7 & 123.962 \\
dih7 & -179.705 \\
oc8 & 1.227201 \\
occ8 & 118.761 \\
dih8 & 171.816 \\
cc9 & 1.532944 \\
ccc9 & 122.407 \\
dih9 & -9.147 \\
cc10 & 1.543893 \\
ccc10 & 112.179 \\
dih10 & -65.059 \\
cc11 & 1.555001 \\
ccc11 & 114.198 \\
dih11 & 64.511 \\
cc12 & 1.551972 \\
ccc12 & 114.051 \\
dih12 & 139.728 \\
oc13 & 1.382847 \\
occ13 & 110.127 \\
dih13 & 163.552 \\
lio14 & 1.606951 \\
lioc14 & 175.194 \\
dih14 & 114.168 \\
cc15 & 1.539439 \\
ccc15 & 111.620 \\
\hline-73.147 \\
1.095413
\end{tabular}




\begin{tabular}{|c|c|}
\hline hcc16 & 102.380 \\
\hline $\operatorname{dih} 16$ & -179.280 \\
\hline hc17 & 1.097225 \\
\hline hcc17 & 110.205 \\
\hline $\operatorname{dih} 17$ & -100.651 \\
\hline hc18 & 1.099414 \\
\hline hcc18 & 111.187 \\
\hline $\operatorname{dih} 18$ & 17.411 \\
\hline hc19 & 1.110346 \\
\hline hcc19 & 107.024 \\
\hline $\operatorname{dih} 19$ & 44.118 \\
\hline hc20 & 1.098300 \\
\hline hec20 & 109.665 \\
\hline $\operatorname{dih} 20$ & -63.407 \\
\hline hc21 & 1.096599 \\
\hline hec 21 & 109.634 \\
\hline $\operatorname{dih} 21$ & 178.054 \\
\hline hc22 & 1.097609 \\
\hline hcc22 & 112.976 \\
\hline $\operatorname{dih} 22$ & 57.428 \\
\hline hc23 & 1.096688 \\
\hline hec23 & 111.220 \\
\hline $\operatorname{dih} 23$ & -55.074 \\
\hline hc24 & 1.095078 \\
\hline hec24 & 112.491 \\
\hline $\operatorname{dih} 24$ & 66.120 \\
\hline hc25 & 1.096329 \\
\hline hcc25 & 109.495 \\
\hline $\operatorname{dih} 25$ & -174.081 \\
\hline hc2 & 1.085371 \\
\hline hec26 & 120.929 \\
\hline $\operatorname{dih} 26$ & 179.675 \\
\hline hc27 & 1.087207 \\
\hline hec 27 & 120.079 \\
\hline $\operatorname{dih} 27$ & -179.984 \\
\hline hc28 & 1.087327 \\
\hline
\end{tabular}


hcc28 120.181

$\operatorname{dih} 28 \quad-179.928$

hc29 1.087212

hcc29 120.134

$\operatorname{dih} 29 \quad-179.755$

hc30 1.082990

hcc30 118.939

$\operatorname{dih} 30 \quad-179.420$

constants

end

Number of imaginary frequency: 0

Thermochemical Data

\section{Vacuum B3LYP Data}

Electronic Energy=

Zero-point correction $=$

Thermal correction to Energy=

Thermal correction to Enthalpy=

Thermal correction to Gibbs Free Energy=

Sum of electronic and zero-point Energies=

Sum of electronic and thermal Energies=

Sum of electronic and thermal Enthalpies=

Sum of electronic and thermal Free Energies=

Thermal translational Energy=

Thermal rotational Energy=

Translational Entropy=

Rotational Entropy=

Corrected Free Energy=

Vacuum MP2 data

Electronic Energy=

IEF-PCM Data $(\varepsilon=2.104)$

$<$ Psi(f)|H+V(f)/2|Psi(f) $>=$
-624.340459 Hartree/Particle

0.246403 Hartree/Particle

0.261854 Hartree/Particle

0.262798 Hartree/Particle

0.201818 Hartree/Particle

-624.094056 Hartree/Particle

-624.078605 Hartree/Particle

-624.077661 Hartree/Particle

-624.138641 Hartree/Particle

$0.889 \mathrm{kcal} / \mathrm{mol}$

$0.889 \mathrm{kcal} / \mathrm{mol}$

$41.756 \quad \mathrm{Cal} / \mathrm{mol}$

$32.438 \quad \mathrm{Cal} / \mathrm{mol}$

-624.106222 Hartree/Particle

-622.304999 Hartree/Particle

-624.361807 Hartree/Particle 
Structure: $3 c$

Z-matrix (molden format)

zmat angstroms

c

c $1 \mathrm{cc} 2$

c $2 \operatorname{cc} 3 \quad 1 \operatorname{ccc} 3$

c $3 \operatorname{cc} 4 \quad 2 \operatorname{ccc} 4 \quad 1 \operatorname{dih} 4$

c $4 \operatorname{cc} 5 \quad 3 \operatorname{ccc} 5 \quad 2$ dih5

c 5 cc6 4 cec6 3 dih 6

$\begin{array}{lll}\text { c } 6 \operatorname{cc} 7 & 5 \operatorname{ccc} 7 & 4 \operatorname{dih} 7\end{array}$

o 7 oc8 6 occ $8 \quad 5 \operatorname{dih} 8$

c $7 \operatorname{cc} 9 \quad 6 \operatorname{ccc} 9 \quad 5$ dih 9

c $9 \operatorname{cc} 10 \quad 7 \operatorname{ccc} 10 \quad 6 \operatorname{dih} 10$

c $9 \operatorname{cc} 11 \quad 7 \operatorname{ccc} 11 \quad 6$ dih 11

c $11 \operatorname{cc} 12 \quad 9 \operatorname{ccc} 12 \quad 7 \operatorname{dih} 12$

o 12 oc13 11 occ13 9 dih13

li 13 lio14 12 lioc14 11 dih14

c $12 \operatorname{cc} 15 \quad 11 \operatorname{ccc} 15 \quad 9 \operatorname{dih} 15$

h 9 hc16 7 hcc16 6 dih16

h 11 hc17 9 hcc17 $\quad 7$ dih17

h 11 hc18 9 hcc18 $\quad 7 \operatorname{dih} 18$

h 12 hc19 11 hcc19 $9 \operatorname{dih} 19$

h 15 hc20 12 hcc20 11 dih20

h 15 hc21 12 hcc21 11 dih 21

h 15 hc22 12 hcc $22 \quad 11 \operatorname{dih} 22$

h 10 hc23 9 hcc $23 \quad 7 \operatorname{dih} 23$

h 10 hc24 9 hcc24 7 dih24

h 10 hc25 9 hcc $25 \quad 7 \operatorname{dih} 25$

h 1 hc26 2 hcc26 $3 \operatorname{dih} 26$

h 2 hc27 3 hcc27 4 dih 27

h 3 hc $28 \quad 2$ hcc $28 \quad 1 \operatorname{dih} 28$

h 4 hc29 3 hcc29 2 dih 29

h 5 hc30 4 hcc30 $3 \operatorname{dih} 30$ 


\begin{tabular}{|c|c|}
\hline variab & \\
\hline $\mathrm{cc} 2$ & 1.395780 \\
\hline $\operatorname{cc} 3$ & 1.397014 \\
\hline $\operatorname{ccc} 3$ & 120.181 \\
\hline $\mathrm{cc} 4$ & 1.399709 \\
\hline $\operatorname{ccc} 4$ & 119.844 \\
\hline $\operatorname{dih} 4$ & 0.379 \\
\hline $\operatorname{cc5} 5$ & 1.391933 \\
\hline $\operatorname{ccc} 5$ & 120.014 \\
\hline $\operatorname{dih} 5$ & 0.144 \\
\hline $\operatorname{cc6}$ & 1.408804 \\
\hline $\operatorname{ccc} 6$ & 120.819 \\
\hline $\operatorname{dih} 6$ & -0.541 \\
\hline $\mathrm{cc} 7$ & 1.497742 \\
\hline $\operatorname{ccc} 7$ & 118.053 \\
\hline $\operatorname{dih} 7$ & 179.798 \\
\hline oc8 & 1.241605 \\
\hline occ 8 & 117.599 \\
\hline $\operatorname{dih} 8$ & -5.084 \\
\hline $\operatorname{cc} 9$ & 1.522645 \\
\hline $\operatorname{ccc} 9$ & 120.527 \\
\hline $\operatorname{dih} 9$ & 176.631 \\
\hline cc10 & 1.551883 \\
\hline $\operatorname{ccc} 10$ & 108.100 \\
\hline $\operatorname{dih} 10$ & 93.023 \\
\hline $\mathrm{cc} 11$ & 1.564682 \\
\hline $\operatorname{ccc} 11$ & 115.248 \\
\hline $\operatorname{dih} 11$ & -138.888 \\
\hline $\mathrm{cc} 12$ & 1.557182 \\
\hline $\operatorname{ccc} 12$ & 118.307 \\
\hline $\operatorname{dih} 12$ & -96.755 \\
\hline oc13 & 1.375318 \\
\hline occ13 & 113.276 \\
\hline $\operatorname{dih} 13$ & 61.697 \\
\hline lio14 & 1.676329 \\
\hline lioc14 & 128.629 \\
\hline
\end{tabular}




\begin{tabular}{|c|c|}
\hline $\operatorname{dih} 14$ & 2.936 \\
\hline $\operatorname{cc} 15$ & 1.543516 \\
\hline $\operatorname{ccc} 15$ & 108.537 \\
\hline $\operatorname{dih} 15$ & -173.907 \\
\hline hc16 & 1.093625 \\
\hline hcc16 & 106.432 \\
\hline $\operatorname{dih} 16$ & -21.454 \\
\hline hc17 & 1.099874 \\
\hline hcc17 & 106.118 \\
\hline $\operatorname{dih} 17$ & 141.418 \\
\hline hc18 & 1.100522 \\
\hline hec18 & 109.230 \\
\hline $\operatorname{dih} 18$ & 27.348 \\
\hline hc19 & 1.113571 \\
\hline hcc19 & 106.529 \\
\hline $\operatorname{dih} 19$ & -60.281 \\
\hline hc20 & 1.095530 \\
\hline hec20 & 108.896 \\
\hline $\operatorname{dih} 20$ & 179.898 \\
\hline hc21 & 1.099966 \\
\hline hec21 & 112.634 \\
\hline $\operatorname{dih} 21$ & 59.408 \\
\hline hc22 & 1.099001 \\
\hline hec22 & 110.293 \\
\hline $\operatorname{dih} 22$ & -62.084 \\
\hline hc23 & 1.095151 \\
\hline hec23 & 109.360 \\
\hline $\operatorname{dih} 23$ & -172.392 \\
\hline hc24 & 1.093485 \\
\hline hec24 & 110.484 \\
\hline $\operatorname{dih} 24$ & 68.769 \\
\hline hc25 & 1.096722 \\
\hline hec25 & 111.623 \\
\hline $\operatorname{dih} 25$ & -52.983 \\
\hline hc26 & 1.084188 \\
\hline $\operatorname{cc} 26$ & 118.629 \\
\hline
\end{tabular}




$\begin{array}{lc}\text { dih26 } & -179.932 \\ \text { hc27 } & 1.086580 \\ \text { hcc27 } & 120.169 \\ \text { dih27 } & -179.781 \\ \text { hc28 } & 1.086970 \\ \text { hcc28 } & 120.046 \\ \text { dih28 } & -179.620 \\ \text { hc29 } & 1.086738 \\ \text { hcc29 } & 120.108 \\ \text { dih29 } & -179.936 \\ \text { hc30 } & 1.084725 \\ \text { hcc30 } & 120.592 \\ \text { dih30 } & 179.740 \\ \text { constants } \\ \text { end }\end{array}$

Number of imaginary frequence: 0

Thermochemical Data 
Vacuum B3LYP Data

Electronic Energy=

-624.367495 Hartree/Particle

Zero-point correction $=$

$0.247094 \quad$ Hartree/Particle

Thermal correction to Energy=

$0.261752 \quad$ Hartree/Particle

Thermal correction to Enthalpy=

0.262697 Hartree/Particle

Thermal correction to Gibbs Free Energy=

$0.204541 \quad$ Hartree/Particle

Sum of electronic and zero-point Energies=

-624.120401 Hartree/Particle

Sum of electronic and thermal Energies=

-624.105743 Hartree/Particle

Sum of electronic and thermal Enthalpies=

-624.104799 Hartree/Particle

Sum of electronic and thermal Free Energies=

$-624.162954$

Hartree/Particle

Thermal translational Energy=

$0.889 \mathrm{kcal} / \mathrm{mol}$

Thermal rotational Energy=

$0.889 \mathrm{kcal} / \mathrm{mol}$

Translational Entropy=

41.756

$\mathrm{Cal} / \mathrm{mol}$

Rotational Entropy=

32.081

$\mathrm{Cal} / \mathrm{mol}$

Corrected Free Energy=

$-624.130705$

Hartree/Particle

Vacuum MP2 data

Electronic Energy=

-622.327986 Hartree/Particle

IEF-PCM Data $(\varepsilon=2.104)$

$<$ Psi(f)|H+V(f)/2|Psi(f) $>=$

-624.380279 Hartree/Particle

\section{Structure 3d}

Z-matrix (molden format)

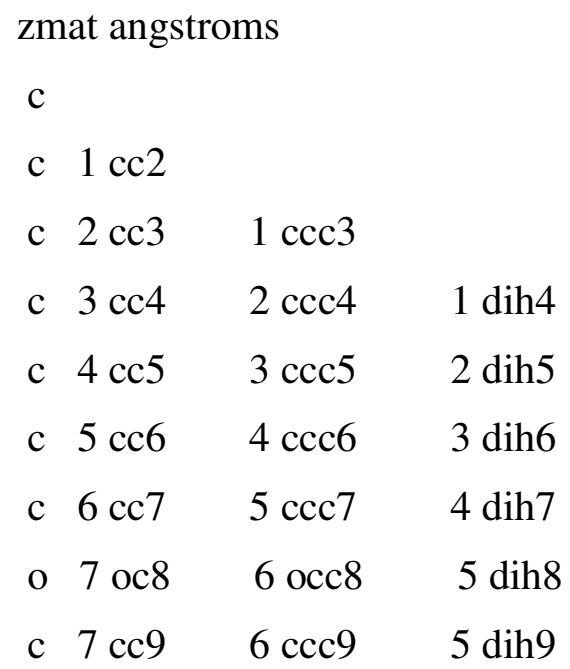




$\begin{array}{ccc}\text { c } 9 \text { cc10 } & 7 \text { ccc10 } & 6 \text { dih10 } \\ \text { c } 10 \text { cc11 } & 9 \text { ccc11 } & 7 \text { dih11 } \\ \text { o } 11 \text { oc12 } & 10 \text { occ12 } & 9 \text { dih12 } \\ \text { c } 9 \text { cc13 } & 7 \text { ccc13 } & 6 \text { dih13 } \\ \text { c } 11 \text { cc14 } & 10 \text { ccc14 } & 9 \text { dih14 } \\ \text { li } 8 \text { lio15 } & 7 \text { lioc15 } & 6 \text { dih } 15 \\ \text { h } 9 \text { hc16 } & 7 \text { hcc16 } & 6 \text { dih16 } \\ \text { h } 10 \text { hc17 } & 9 \text { hcc17 } & 7 \text { dih17 } \\ \text { h } 10 \text { hc18 } & 9 \text { hcc18 } & 7 \text { dih18 } \\ \text { h } 11 \text { hc19 } & 10 \text { hcc19 } & 9 \text { dih19 } \\ \text { h } 14 \text { hc20 } & 11 \text { hcc20 } & 10 \text { dih20 } \\ \text { h } 14 \text { hc21 } & 11 \text { hcc21 } & 10 \operatorname{dih} 21 \\ \text { h } 14 \text { hc22 } & 11 \text { hcc22 } & 10 \operatorname{dih} 22 \\ \text { h } 13 \text { hc23 } & 9 \text { hcc23 } & 7 \text { dih23 } \\ \text { h } 13 \text { hc24 } & 9 \text { hcc24 } & 7 \text { dih24 } \\ \text { h } 13 \text { hc25 } & 9 \text { hcc25 } & 7 \text { dih25 } \\ \text { h } 1 \text { hc26 } & 2 \text { hcc26 } & 3 \operatorname{dih} 26 \\ \text { h } 2 \text { hc27 } & 3 \text { hcc27 } & 4 \operatorname{dih} 27 \\ \text { h } 3 \text { hc28 } & 2 \text { hcc28 } & 1 \text { dih28 } \\ \text { h } 4 \text { hc29 } & 3 \text { hcc29 } & 2 \operatorname{dih} 29 \\ \text { h } 5 \text { hc30 } & 4 \text { hcc30 } & 3 \operatorname{dih} 30 \\ \text { variables } & & \end{array}$

$\begin{array}{ll}\text { cc2 } & 1.391348 \\ \text { cc3 } & 1.400088 \\ \text { ccc3 } & 119.982 \\ \text { cc4 } & 1.397785 \\ \text { ccc4 } & 120.015 \\ \text { dih4 } & -0.340 \\ \text { cc5 } & 1.394597 \\ \text { ccc5 } & 120.133 \\ \text { dih5 } & -0.586 \\ \text { cc6 } & 1.408181 \\ \text { ccc6 } & 120.386 \\ \text { dih6 } & 0.877 \\ \text { cc7 } & 1.483610 \\ \text { ccc7 } & 122.674\end{array}$




$\begin{array}{ll}\text { dih7 } & 178.425 \\ \text { oc8 } & 1.252786 \\ \text { occ8 } & 118.478 \\ \text { dih8 } & -168.025 \\ \text { cc9 } & 1.512134 \\ \text { ccc9 } & 122.244 \\ \text { dih9 } & 12.491 \\ \text { cc10 } & 1.579637 \\ \text { ccc10 } & 112.747 \\ \text { dih10 } & -104.648 \\ \text { cc11 } & 1.577663 \\ \text { ccc11 } & 125.844 \\ \text { dih11 } & -52.720 \\ \text { oc12 } & 1.307625 \\ \text { occ12 } & 110.291 \\ \text { dih12 } & 178.297 \\ \text { cc13 } & 1.545011 \\ \text { ccc13 } & 110.226 \\ \text { dih13 } & 126.156 \\ \text { cc14 } & 1.591396 \\ \text { ccc14 } & 113.570 \\ \text { dih14 } & -57.716 \\ \text { lio15 } & 1.821164 \\ \text { lioc15 } & 118.578 \\ \text { dih15 } & 136.177 \\ \text { hc16 } & 1.093673 \\ \text { hcc16 } & 108.259 \\ \text { dih16 } & 10.834 \\ \text { hc17 } & 1.095871 \\ \text { hcc17 } & 108.028 \\ \text { dih17 } & 69.941 \\ \text { hc18 } & 1.098212 \\ \text { hcc18 } & 106.071 \\ \text { dih18 } & -176.621 \\ \text { hc19 } & 1.180391 \\ \text { hcc19 } & 104.656\end{array}$




\begin{tabular}{lc} 
dih19 & 59.255 \\
hc20 & 1.093739 \\
hcc20 & 106.298 \\
dih20 & -65.990 \\
hc21 & 1.097709 \\
hcc21 & 108.052 \\
dih21 & 179.494 \\
hc22 & 1.115221 \\
hcc22 & 120.625 \\
dih22 & 52.589 \\
hc23 & 1.096734 \\
hcc23 & 110.852 \\
dih23 & -62.330 \\
hc24 & 1.092216 \\
hcc24 & 113.119 \\
dih24 & 58.529 \\
hc25 & 1.095174 \\
hih30 & 179.494 \\
end 25 & 109.209 \\
dih25 & 178.752 \\
hc26 & 1.084857 \\
hcc26 & 120.657 \\
dih26 & -179.471 \\
hc27 & 1.086603 \\
hcc27 & 120.100 \\
dih27 & 179.945 \\
hc28 & 1.086895 \\
hcc28 & 120.023 \\
hih28 & 179.575 \\
hcc & 1.086353 \\
\hline
\end{tabular}


Number of imaginary frequency: 0

Thermochemical Data

Vacuum B3LYP Data

Electronic Energy=

Zero-point correction $=$

Thermal correction to Energy=

Thermal correction to Enthalpy=

Thermal correction to Gibbs Free Energy=

Sum of electronic and zero-point Energies=

Sum of electronic and thermal Energies=

Sum of electronic and thermal Enthalpies=

Sum of electronic and thermal Free Energies=

Thermal translational Energy=

Thermal rotational Energy=

Translational Entropy=

Rotational Entropy=

Corrected Free Energy=

Vacuum MP2 data

Electronic Energy=

IEF-PCM Data $(\varepsilon=2.104)$

$<$ Psi(f)|H+V(f)/2|Psi(f) $>=$

Structure: $4 \mathrm{a}$

Z-matrix (molden format)

zmat angstroms

c

c $1 \mathrm{cc} 2$

c $2 \operatorname{cc} 3 \quad 1 \operatorname{ccc} 3$

c $3 \operatorname{cc} 4 \quad 2 \operatorname{ccc} 4 \quad 1 \operatorname{dih} 4$
-624.291290 Hartree/Particle

0.243820 Hartree/Particle

0.258749 Hartree/Particle

0.259693 Hartree/Particle

0.200831 Hartree/Particle

-624.047470 Hartree/Particle

-624.032542 Hartree/Particle

$-624.031597 \quad$ Hartree/Particle

-624.090459 Hartree/Particle

$0.889 \mathrm{kcal} / \mathrm{mol}$

$0.889 \mathrm{kcal} / \mathrm{mol}$

$41.756 \quad \mathrm{Cal} / \mathrm{mol}$

$32.148 \quad \mathrm{Cal} / \mathrm{mol}$

-624.058178 Hartree/Particle

-622.247533 Hartree/Particle

-624.313129 Hartree/Particle 


\begin{tabular}{|c|c|c|c|}
\hline \multicolumn{2}{|c|}{ c $4 \operatorname{cc} 5$} & $3 \operatorname{ccc} 5$ & $2 \operatorname{dih} 5$ \\
\hline \multicolumn{2}{|c|}{ c 5 cc6 } & $4 \operatorname{ccc} 6$ & 3 dih 6 \\
\hline \multicolumn{2}{|c|}{ c $2 \mathrm{cc} 7$} & $3 \operatorname{ccc} 7$ & $4 \operatorname{dih} 7$ \\
\hline \multicolumn{2}{|c|}{ o 7 oc 8} & 2 occ 8 & $3 \operatorname{dih} 8$ \\
\hline \multicolumn{2}{|c|}{ c $7 \mathrm{cc} 9$} & $2 \operatorname{ccc} 9$ & $3 \operatorname{dih} 9$ \\
\hline \multicolumn{2}{|c|}{ c $9 \operatorname{cc} 10$} & $7 \operatorname{ccc} 10$ & $2 \operatorname{dih} 10$ \\
\hline \multicolumn{2}{|c|}{ c $9 \mathrm{cc} 11$} & $7 \operatorname{ccc} 11$ & 8 dih11 \\
\hline \multicolumn{2}{|c|}{ c $11 \mathrm{cc} 12$} & $9 \operatorname{ccc} 12$ & $7 \operatorname{dih} 12$ \\
\hline \multicolumn{2}{|c|}{ о 12 oc13 } & 11 occ13 & $9 \operatorname{dih} 13$ \\
\hline \multicolumn{2}{|c|}{ li 13 lio14 } & 12 lioc14 & $11 \operatorname{dih} 14$ \\
\hline \multicolumn{2}{|c|}{ c $12 \mathrm{cc} 15$} & $11 \operatorname{ccc} 15$ & $9 \operatorname{dih} 15$ \\
\hline \multicolumn{2}{|c|}{ h 12 hc16 } & 11 hcc16 & $9 \operatorname{dih} 16$ \\
\hline \multicolumn{2}{|c|}{ h 3 hc17 } & 2 hcc17 & $7 \operatorname{dih} 17$ \\
\hline \multicolumn{2}{|c|}{ h 4 hc18 } & 3 hcc18 & $2 \operatorname{dih} 18$ \\
\hline \multicolumn{2}{|c|}{ h 5 hc19 } & 4 hcc19 & $3 \operatorname{dih} 19$ \\
\hline \multicolumn{2}{|c|}{ h 6 hc20 } & 5 hcc 20 & $4 \operatorname{dih} 20$ \\
\hline \multicolumn{2}{|c|}{ h 1 hc21 } & 2 hcc21 & $7 \operatorname{dih} 21$ \\
\hline \multicolumn{2}{|c|}{ h 9 hc22 } & 11 hcc 22 & $12 \operatorname{dih} 22$ \\
\hline \multicolumn{2}{|c|}{ h 10 hc 23} & 9 hcc 23 & $11 \operatorname{dih} 23$ \\
\hline \multicolumn{2}{|c|}{ h 10 hc 24} & 9 hcc24 & $11 \operatorname{dih} 24$ \\
\hline \multicolumn{2}{|c|}{ h 10 hc 25} & 9 hcc 25 & $11 \operatorname{dih} 25$ \\
\hline \multicolumn{2}{|c|}{ h 15 hc 26} & 12 hcc 26 & $11 \operatorname{dih} 26$ \\
\hline \multicolumn{2}{|c|}{ h 15 hc 27} & 12 hcc 27 & $11 \operatorname{dih} 27$ \\
\hline \multicolumn{2}{|c|}{ h 15 hc 28} & 12 hcc 28 & $11 \operatorname{dih} 28$ \\
\hline \multicolumn{2}{|c|}{ h 11 hc 29} & 9 hcc 29 & $7 \operatorname{dih} 29$ \\
\hline \multicolumn{2}{|c|}{ h 11 hc30 } & 9 hcc30 & $7 \operatorname{dih} 30$ \\
\hline \multicolumn{4}{|c|}{ variables } \\
\hline $\mathrm{cc}^{2}$ & \multicolumn{3}{|c|}{1.408031} \\
\hline $\mathrm{cc}^{3}$ & \multicolumn{3}{|c|}{1.407035} \\
\hline $\mathrm{ccc}$ & 11 & 3.702 & \\
\hline $\mathrm{cc}$ & 1.3 & 95343 & \\
\hline $\mathrm{ccc}$ & & .523 & \\
\hline .11 & & 048 & \\
\hline $\operatorname{ccs}$ & 1.3 & 97484 & \\
\hline & & .141 & \\
\hline & & & \\
\hline
\end{tabular}




$\begin{array}{ll}\text { cc6 } & 1.399478 \\ \text { ccc6 } & 119.893 \\ \text { dih6 } & 0.337 \\ \text { cc7 } & 1.496557 \\ \text { ccc7 } & 123.075 \\ \text { dih7 } & -179.584 \\ \text { oc8 } & 1.243073 \\ \text { occ8 } & 118.035 \\ \text { dih8 } & 175.790 \\ \text { cc9 } & 1.523716 \\ \text { ccc9 } & 120.185 \\ \text { dih9 } & -5.513 \\ \text { cc10 } & 1.537566 \\ \text { ccc10 } & 111.637 \\ \text { dih10 } & -145.069 \\ \text { cc11 } & 1.573482 \\ \text { ccc11 } & 111.821 \\ \text { dih11 } & -97.188 \\ \text { cc12 } & 1.569869 \\ \text { ccc12 } & 118.840 \\ \text { dih12 } & 82.602 \\ \text { oc13 } & 1.371778 \\ \text { occ13 } & 113.086 \\ \text { dih13 } & -41.555 \\ \text { lio14 } & 1.663655 \\ \text { lioc14 } & 141.405 \\ \text { dih14 } & 21.306 \\ \text { cc15 } & 1.546488 \\ \text { ccc15 } & 112.612 \\ \text { dih15 } & 86.695 \\ \text { hc16 } & 1.113788 \\ \text { hcc16 } & 103.028 \\ \text { dih16 } & -160.982 \\ \text { hc17 } & 1.084396 \\ \text { hcc17 } & 120.671 \\ \text { dih17 } & -0.285 \\ & \end{array}$




$\begin{array}{ll}\text { hc18 } & 1.086587 \\ \text { hcc18 } & 119.686 \\ \text { dih18 } & 179.605 \\ \text { hc19 } & 1.087011 \\ \text { hcc19 } & 120.022 \\ \text { dih19 } & -179.656 \\ \text { hc20 } & 1.086752 \\ \text { hcc20 } & 120.105 \\ \text { dih20 } & -179.940 \\ \text { hc21 } & 1.084874 \\ \text { hcc21 } & 118.602 \\ \text { dih21 } & -0.302 \\ \text { hc22 } & 1.096549 \\ \text { hcc22 } & 105.288 \\ \text { dih22 } & -163.290 \\ \text { hc23 } & 1.096813 \\ \text { hcc23 } & 110.795 \\ \text { dih23 } & -176.498 \\ \text { hc24 } & 1.095080 \\ \text { hcc24 } & 109.563 \\ \text { dih24 } & -57.511 \\ \text { hc25 } & 1.091444 \\ \text { hcc25 } & 112.085 \\ \text { dih25 } & 62.045 \\ \text { hc26 } & 1.096144 \\ \text { hcc26 } & 108.411 \\ \text { dih26 } & 173.489 \\ \text { hc27 } & 1.095372 \\ \text { hcc27 } & 112.105 \\ \text { dih27 } & -68.004 \\ \text { hc28 } & 1.100038 \\ \text { hcc28 } & 111.985 \\ \text { dih28 } & 54.233 \\ \text { hc29 } & 1.100241 \\ \text { hcc29 } & 106.853 \\ \text { dih29 } & -152.979 \\ & \end{array}$


hc30 $\quad 1.098121$

hcc30 107.430

$\operatorname{dih} 30 \quad-39.524$

constants

end

Number of imaginary frequency: 0

Thermochemical Data

\section{Vacuum B3LYP Data}

Electronic Energy=

Zero-point correction $=$

Thermal correction to Energy=

Thermal correction to Enthalpy=

Thermal correction to Gibbs Free Energy=

Sum of electronic and zero-point Energies=

Sum of electronic and thermal Energies=

Sum of electronic and thermal Enthalpies=

Sum of electronic and thermal Free Energies=

Thermal translational Energy=

Thermal rotational Energy=

Translational Entropy=

Rotational Entropy=

Corrected Free Energy=

Vacuum MP2 data

Electronic Energy=

IEF-PCM Data $(\varepsilon=2.104)$

$<\operatorname{Psi}(\mathrm{f})|\mathrm{H}+\mathrm{V}(\mathrm{f}) / 2| \operatorname{Psi}(\mathrm{f})>=$

Structure: $4 b$

Z-matrix (molden format)
-624.362428 Hartree/Particle

0.247285 Hartree/Particle

0.261838 Hartree/Particle

0.262782 Hartree/Particle

0.205309 Hartree/Particle

-624.116143 Hartree/Particle

$-624.101590 \quad$ Hartree/Particle

-624.100646 Hartree/Particle

-624.158118 Hartree/Particle

$0.889 \mathrm{kcal} / \mathrm{mol}$

$0.889 \mathrm{kcal} / \mathrm{mol}$

$41.756 \quad \mathrm{Cal} / \mathrm{mol}$

$32.003 \quad \mathrm{Cal} / \mathrm{mol}$

-624.125906 Hartree/Particle

$-622.325001 \quad$ Hartree/Particle

-624.375798 Hartree/Particle 


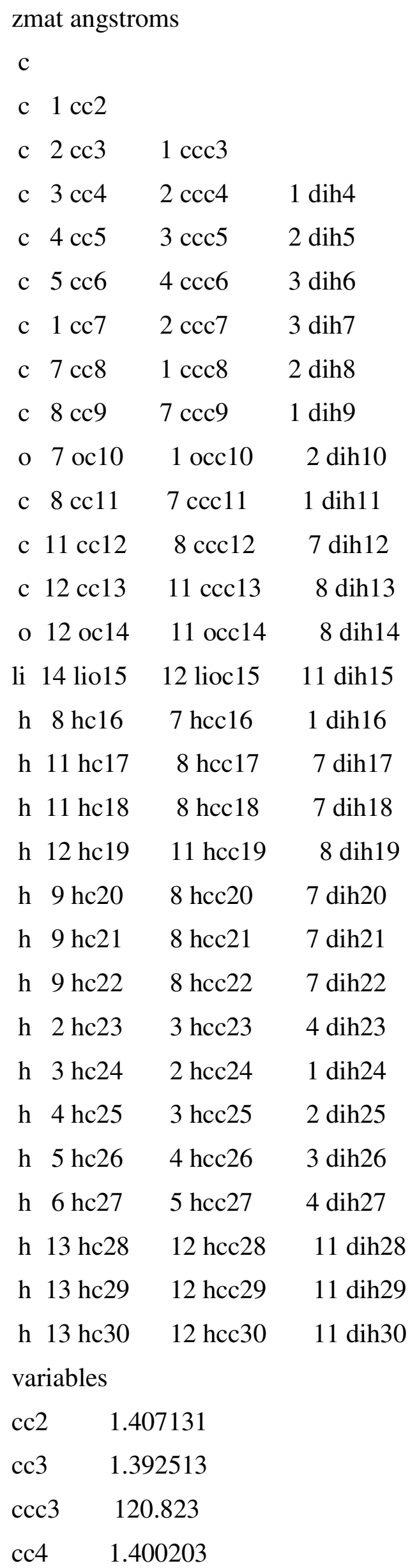




\begin{tabular}{|c|c|}
\hline $\operatorname{ccc} 4$ & 120.052 \\
\hline $\operatorname{dih} 4$ & -0.414 \\
\hline $\operatorname{cc} 5$ & 1.396429 \\
\hline $\operatorname{ccc} 5$ & 119.748 \\
\hline $\operatorname{dih} 5$ & 0.109 \\
\hline $\operatorname{cc} 6$ & 1.397612 \\
\hline $\operatorname{ccc} 6$ & 120.158 \\
\hline $\operatorname{dih} 6$ & 0.277 \\
\hline $\operatorname{cc} 7$ & 1.507049 \\
\hline $\operatorname{ccc} 7$ & 117.645 \\
\hline $\operatorname{dih} 7$ & 179.518 \\
\hline $\operatorname{cc} 8$ & 1.532599 \\
\hline $\operatorname{ccc} 8$ & 121.260 \\
\hline $\operatorname{dih} 8$ & 176.142 \\
\hline $\operatorname{cc} 9$ & 1.545230 \\
\hline $\operatorname{ccc} 9$ & 108.140 \\
\hline dih9 & 108.025 \\
\hline oc 10 & 1.226342 \\
\hline occ10 & 119.312 \\
\hline $\operatorname{dih} 10$ & -5.824 \\
\hline $\operatorname{cc} 11$ & 1.544170 \\
\hline $\operatorname{ccc} 11$ & 110.734 \\
\hline $\operatorname{dih} 11$ & -130.051 \\
\hline $\operatorname{cc} 12$ & 1.547431 \\
\hline $\operatorname{ccc} 12$ & 116.361 \\
\hline $\operatorname{dih} 12$ & 67.542 \\
\hline $\operatorname{cc} 13$ & 1.540237 \\
\hline $\operatorname{ccc} 13$ & 112.049 \\
\hline $\operatorname{dih} 13$ & 67.470 \\
\hline oc14 & 1.383281 \\
\hline occ14 & 109.883 \\
\hline $\operatorname{dih} 14$ & -169.357 \\
\hline lio15 & 1.605432 \\
\hline lioc 15 & 176.337 \\
\hline $\operatorname{dih} 15$ & 46.228 \\
\hline hc16 & 1.093531 \\
\hline
\end{tabular}




$\begin{array}{ll}\text { hcc16 } & 110.163 \\ \text { dih16 } & -9.642 \\ \text { hc17 } & 1.100268 \\ \text { hcc17 } & 108.675 \\ \text { dih17 } & -170.075 \\ \text { hc18 } & 1.096105 \\ \text { hcc18 } & 108.376 \\ \text { dih18 } & -53.839 \\ \text { hc19 } & 1.109963 \\ \text { hcc19 } & 107.357 \\ \text { dih19 } & -50.021 \\ \text { hc20 } & 1.097408 \\ \text { hcc20 } & 111.849 \\ \text { dih20 } & -58.857 \\ \text { hc21 } & 1.095271 \\ \text { hcc21 } & 110.299 \\ \text { dih21 } & 61.471 \\ \text { hc22 } & 1.096319 \\ \text { hcc22 } & 110.034 \\ \text { dih22 } & -178.866 \\ \text { hc23 } & 1.085395 \\ \text { hcc23 } & 120.997 \\ \text { dih23 } & 179.642 \\ \text { hc24 } & 1.087191 \\ \text { hcc24 } & 119.898 \\ \text { dih24 } & 179.664 \\ \text { hc25 } & 1.087281 \\ \text { hcc25 } & 120.161 \\ \text { dih25 } & -179.886 \\ \text { hc26 } & 1.087046 \\ \text { hcc26 } & 120.159 \\ \text { dih26 } & -179.862 \\ \text { hc27 } & 1.084689 \\ \text { hcc27 } & 118.735 \\ \text { dih27 } & 179.981 \\ \text { hc28 } & 1.096370 \\ & \end{array}$




$\begin{array}{ll}\text { hcc28 } & 109.574 \\ \text { dih28 } & 179.024 \\ \text { hc29 } & 1.098494 \\ \text { hcc29 } & 112.816 \\ \text { dih29 } & -60.796 \\ \text { hc30 } & 1.098707 \\ \text { hcc30 } & 109.970 \\ \text { dih30 } & 60.485 \\ \text { constants } \\ \text { end }\end{array}$

Number of imaginary frequency: 0

Thermochemical Data

Vacuum B3LYP Data

Electronic Energy=

Zero-point correction=

-624.344922 Hartree/Particle

Thermal correction to Energy=

0.246366 Hartree/Particle

Thermal correction to Enthalpy=

0.261794 Hartree/Particle

Thermal correction to Gibbs Free Energy=

0.262739 Hartree/Particle

Sum of electronic and zero-point Energies=

0.201042 Hartree/Particle

Sum of electronic and thermal Energies=

-624.098556 Hartree/Particle

Sum of electronic and thermal Enthalpies=

-624.083128 Hartree/Particle

Sum of electronic and thermal Free Energies= -624.082183 Hartree/Particle

Thermal translational Energy=

$-624.143880$

Hartree/Particle

Thermal rotational Energy=

0.889

$\mathrm{kcal} / \mathrm{mol}$

$0.889 \quad \mathrm{kcal} / \mathrm{mol}$

Translational Entropy=

41.756

$\mathrm{Cal} / \mathrm{mol}$

Rotational Entropy=

32.365

$\mathrm{Cal} / \mathrm{mol}$

Corrected Free Energy=

$-624.111496$

Hartree/Particle

Vacuum MP2 data

Electronic Energy=

-622.309946 Hartree/Particle

IEF-PCM Data ( $\varepsilon=2.104)$

$<\operatorname{Psi}(\mathrm{f})|\mathrm{H}+\mathrm{V}(\mathrm{f}) / 2| \operatorname{Psi}(\mathrm{f})>=$

$-624.365863 \quad$ Hartree/Particle 
Structure: $4 c$

Z-matrix (molden format)

zmat angstroms

c

c $1 \mathrm{cc} 2$

c $2 \operatorname{cc} 3 \quad 1 \operatorname{ccc} 3$

c $3 \operatorname{cc} 4 \quad 2 \operatorname{ccc} 4 \quad 1 \operatorname{dih} 4$

c $4 \operatorname{cc} 5 \quad 3 \operatorname{ccc} 5 \quad 2$ dih5

c 5 cc6 4 cec6 3 dih 6

$\begin{array}{lll}\text { c } 1 \operatorname{cc} 7 & 2 \operatorname{ccc} 7 & 3 \operatorname{dih} 7\end{array}$

o 7 oc8 1 occ $8 \quad 2$ dih8

c $7 \operatorname{cc} 9 \quad 1 \operatorname{ccc} 9 \quad 2 \operatorname{dih} 9$

c $9 \operatorname{cc} 10 \quad 7 \operatorname{ccc} 10 \quad 1 \operatorname{dih} 10$

c $10 \operatorname{cc} 11 \quad 9 \operatorname{ccc} 11 \quad 7$ dih11

o 11 oc12 10 occ12 $9 \operatorname{dih} 12$

c $9 \operatorname{cc} 13 \quad 7 \operatorname{ccc} 13 \quad 1 \operatorname{dih} 13$

c 11 cc14 $10 \operatorname{ccc} 14 \quad 9 \operatorname{dih} 14$

li 8 lio15 7 lioc15 1 dih15

h 9 hc16 7 hcc16 1 dih16

h 13 hc17 9 hcc17 $\quad 7$ dih17

h 13 hc18 9 hcc18 $\quad 7$ dih 18

h 13 hc19 9 hcc19 7 dih19

h 2 hc20 3 hec20 4 dih 20

h 3 hc21 2 hcc21 1 dih21

h 4 hc22 3 hcc22 2 dih 22

h 5 hc23 4 hcc23 3 dih 23

h 6 hc24 5 hcc24 4 dih24

h 10 hc25 9 hcc $25 \quad 7 \operatorname{dih} 25$

h 10 hc26 9 hcc26 7 dih26

h 11 hc27 10 hcc27 $9 \operatorname{dih} 27$

h 14 hc28 11 hcc28 $10 \operatorname{dih} 28$

h 14 hc29 11 hcc29 $10 \operatorname{dih} 29$

h 14 hc30 11 hcc30 $10 \operatorname{dih} 30$ 


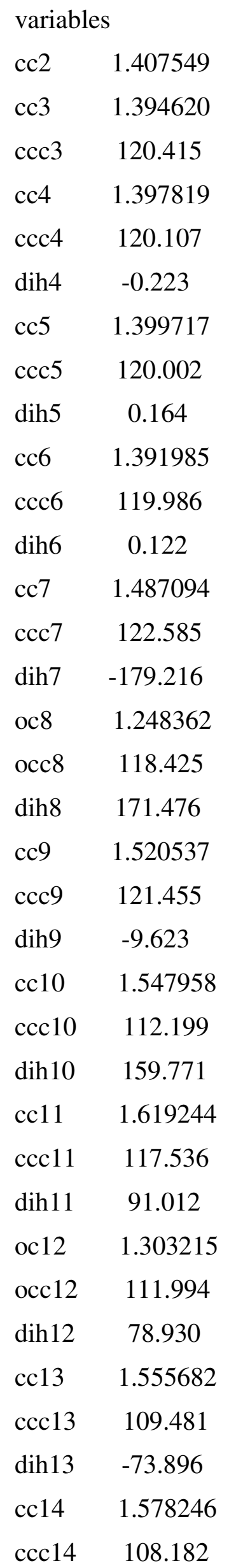




$\begin{array}{ll}\text { dih14 } & -155.292 \\ \text { lio15 } & 1.842024 \\ \text { lioc15 } & 117.831 \\ \text { dih15 } & 178.333 \\ \text { hc16 } & 1.098107 \\ \text { hcc16 } & 106.947 \\ \text { dih16 } & 41.782 \\ \text { hc17 } & 1.092086 \\ \text { hcc17 } & 108.867 \\ \text { dih17 } & -176.363 \\ \text { hc18 } & 1.095117 \\ \text { hcc18 } & 111.216 \\ \text { dih18 } & 62.018 \\ \text { hc19 } & 1.093102 \\ \text { hcc19 } & 111.232 \\ \text { dih19 } & -59.477 \\ \text { hc20 } & 1.084520 \\ \text { hcc20 } & 118.993 \\ \text { dih20 } & -179.876 \\ \text { hc21 } & 1.086430 \\ \text { hcc21 } & 119.725 \\ \text { dih21 } & 179.765 \\ \text { hc22 } & 1.086918 \\ \text { hcc22 } & 119.962 \\ \text { dih22 } & -179.861 \\ \text { hc23 } & 1.086596 \\ \text { hcc23 } & 120.107 \\ \text { dih23 } & -179.895 \\ \text { hc24 } & 1.084917 \\ \text { hcc24 } & 120.677 \\ \text { dih24 } & 179.890 \\ \text { hc25 } & 1.110479 \\ \text { hcc25 } & 108.477 \\ \text { dih25 } & -41.888 \\ \text { hc26 } & 1.093218 \\ \text { hcc26 } & 108.699 \\ & \end{array}$




$\begin{array}{ll}\operatorname{dih} 26 & -155.288 \\ \text { hc27 } & 1.178723 \\ \text { hcc27 } & 107.138 \\ \operatorname{dih} 27 & -42.894 \\ \text { hc28 } & 1.095981 \\ \text { hcc28 } & 108.866 \\ \text { dih28 } & -59.747 \\ \text { hc29 } & 1.093373 \\ \text { hcc29 } & 107.896 \\ \text { dih29 } & -177.577 \\ \text { hc30 } & 1.118659 \\ \text { hcc30 } & 117.560 \\ \text { dih30 } & 61.141 \\ \text { constants } \\ \text { end }\end{array}$

Number of imaginary frequency: 0

Thermochemical Data 
Vacuum B3LYP Data

Electronic Energy=

Zero-point correction=

Thermal correction to Energy=

Thermal correction to Enthalpy=

Thermal correction to Gibbs Free Energy=

Sum of electronic and zero-point Energies=

Sum of electronic and thermal Energies=

Sum of electronic and thermal Enthalpies=

Sum of electronic and thermal Free Energies=

Thermal translational Energy=

Thermal rotational Energy=

Translational Entropy=

Rotational Entropy=

Corrected Free Energy=

Vacuum MP2 data

Electronic Energy=

IEF-PCM Data $(\varepsilon=2.104)$

$<\operatorname{Psi}(\mathrm{f})|\mathrm{H}+\mathrm{V}(\mathrm{f}) / 2| \mathrm{Psi}(\mathrm{f})>=$
-624.298762 Hartree/Particle

0.243917 Hartree/Particle

0.258663 Hartree/Particle

0.259607 Hartree/Particle

0.202357 Hartree/Particle

-624.054845 Hartree/Particle

-624.040099 Hartree/Particle

-624.039155 Hartree/Particle

-624.096405 Hartree/Particle

$0.889 \mathrm{kcal} / \mathrm{mol}$

$0.889 \quad \mathrm{kcal} / \mathrm{mol}$

$41.756 \quad \mathrm{Cal} / \mathrm{mol}$

$32.063 \quad \mathrm{Cal} / \mathrm{mol}$

-624.064165 Hartree/Particle

-622.257665 Hartree/Particle

-624.317067 Hartree/Particle 